\title{
Events in Time: Basic Analysis of Poisson Data
}

M. E. Engelhardt

Published September 1994

\section{Idaho National Engineering Laboratory \\ EG\&G Idaho, Inc. \\ Idaho Falls, Idaho $\mathbf{8 3 4 1 5}$}

Prepared for the

U.S. Department of Energy

Reliability and Risk Analysis Branch

Safety Programs Division

Office for Analysis and Evaluation of Operational Data

U.S. Nuclear Regulatory Commission

Washington, DC 20555

Under DOE Idaho Operations Office

Contract DE-AC07-76ID01570

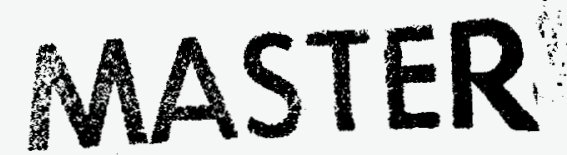

FIN E8205 


\section{DISCLAIMER}

This report was prepared as an account of work sponsored by an agency of the United States Government. Neither the United States Government nor any agency thereof, nor any of their employees, make any warranty, express or implied, or assumes any legal liability or responsibility for the accuracy, completeness, or usefulness of any information, apparatus, product, or process disciosed, or represents that its use would not infringe privately owned rights. Reference herein to any specific commercial product, process, or service by trade name, trademark, manufacturer, or otherwise does not necessarily constitute or imply its endorsement, recommendation, or favoring by the United States Government or any agency thereof. The views and opinions of authors expressed herein do not necessarily state or reflect those of the United States Government or any agency thereof. 


\section{DISCLAIMER}

Portions of this document may be illegible in electronic image products. Images are produced from the best available original document. 


\section{ABSTRACT}

This report presents basic statistical methods for analyzing Poisson data, such as the number of events in some period of time. It gives point estimates, confidence intervals, and Bayesian intervals for the rate of occurrence per unit of time. It shows how to compare subsets of the data, both graphically and by statistical tests, and how to look for trends in time. It presents a compound model when the rate of occurrence varies randomly. Examples and SAS programs are given. 


\section{SUMMARY}

This report deals primarily with statistical methods for analyzing data based on counts of events $n$ during time periods of fixed length $t$. Common examples are observed failure counts $n_{\mathrm{i}}$ of components or systems in disjoint time intervals of lengths $t_{\mathrm{i}}$. Although the main focus is on methods which apply with count-data, some analyses for exact event-time data are also discussed.

First, the simplest case: Consider events which occur independently in some fixed time interval, with a constant rate of occurrence $\lambda$ per unit of time. Formulas are given for the point estimate (maximum likelihood estimate) and confidence interval for $\lambda$, and the Bayes posterior distribution is given, based on a noninformative prior distribution.

The assumption of a constant rate $\lambda$ can be investigated in several ways. When there are natural subsets of the data, such as counts in different time intervals, or counts from different plants during the same time interval, graphical methods and statistical tests are given to investigate whether $\lambda$ is the same for all the subsets. The graphical methods use confidence intervals plotted side by side, and the tests use contingency tables with one row for the counts from the different sets of data. When the individual events are arranged in chronological order, a cumulative plot of the event count helps show whether $\lambda$ is constant throughout the sequence. Two trend tests, one attributed to Laplace and the other to Mann, are considered to test the assumption of constant rate against the alternative that $\lambda$ is increasing with time. These tests, suitably modified, can also test for a decreasing trend, which is what one might expect when newer equipment or improved maintenance procedures are introduced. A test based on counting the number of runs is considered for testing whether events are occurring independently.

A compound model is presented to model the case when the data come from a number of distinct sources and $\lambda$ varies among the sources. Here $\lambda$ is assumed to follow a gamma distribution, whose parameters are estimated from the data. The value of $\lambda_{i}$ for the $i$ th data source is then estimated by the empirical Bayes method. 


\section{FORWARD}

The Office for Analysis and Evaluation of Operational Data (AEOD) of the U.S. Nuclear Regulatory Commission (NRC) is continually reviewing data from U.S. commercial nuclear power plants. Statistical analysis forms an important part of this work. Accordingly, the Reliability and Risk Analysis Branch of AEOD has asked the Idaho National Engineering Laboratory (INEL) to write a series of reports presenting the appropriate statistical tools for the kinds of data most commonly encountered. These reports are being written in parallel with a series of studies on the performance of safety systems in nuclear power plants, and reflect the influence of those studies.

The reports are expected to provide tools and guidance to analysts of NRC data, although the statistical methods can, by their nature, be applied to data from many other fields. The reports are intended to quickly help a new user. For example, the reports on statistical methods should be readable and immediately usable by a person with training in statistics but with no experience analyzing such data. The report on collecting operational data should be understandable by anyone with a technical background, although the examples are slanted towards an engineer with nuclear experience. In addition, the reports should be usable as texts or references in short courses for persons with less training.

The first three reports in this series, appearing in September, 1994, are

Collecting Operational Event Data for Statistical Analysis, EGG-RAAM-11086, by Corwin L. Atwood

Hits per Trial: Basic Analysis of Binomial Data, EGG-RAAM-11041, by Corwin L. atwood Events in Time: Basic Analysis of Poisson Data, EGG-RAAM-11088, by M. E. Engelhardt

Other reports in the series are planned for 1995. 


\section{ACKNOWLEDGMENTS}

Mark DeHaan and Cory Atwood contributed the SAS programs. Helpful review comments were provided by Cory Atwood, Cindy Gentillon, and Dana Kelly. Harry Martz suggested the Kass-Steffey adjustment to the empirical Bayes intervals, and helped improve the overall presentation. 


\section{CONTENTS}

ABSTRACT $\ldots \ldots \ldots \ldots \ldots \ldots \ldots \ldots \ldots \ldots \ldots \ldots \ldots \ldots \ldots$ iii

SUMMARY $\ldots \ldots \ldots \ldots \ldots \ldots \ldots \ldots \ldots \ldots \ldots \ldots \ldots \ldots \ldots \ldots$

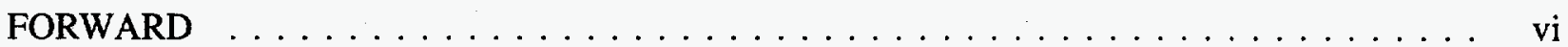

ACKNOWLEDGMENTS $\ldots \ldots \ldots \ldots \ldots \ldots \ldots \ldots \ldots \ldots \ldots \ldots \ldots$ vii

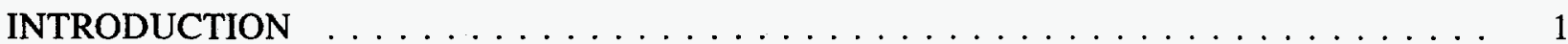

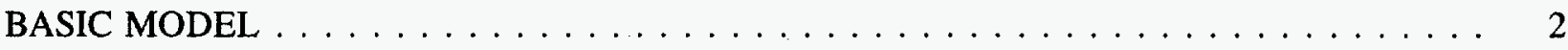

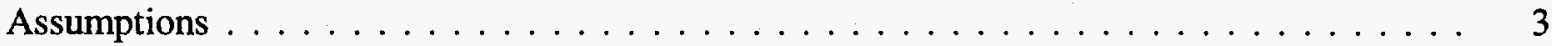

Point Estimation $\ldots \ldots \ldots \ldots \ldots \ldots \ldots \ldots \ldots \ldots \ldots \ldots$

Standard Deviation of Estimator $\ldots \ldots \ldots \ldots \ldots \ldots$

Confidence Interval for $\lambda \ldots \ldots \ldots \ldots \ldots \ldots \ldots \ldots$

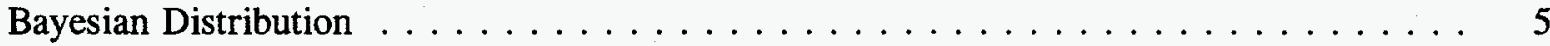

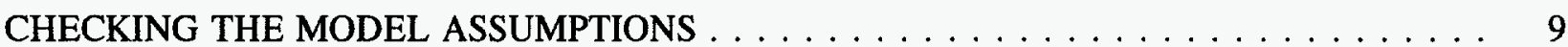

Assumption of Same $\lambda$ in Different Subsets $\ldots \ldots \ldots \ldots \ldots \ldots$

Graphical Techniques . . . . . . . . . . . . . . . . . . . . . . 10

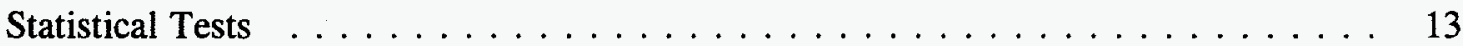

Assumption of Constant $\lambda$ for Events in Sequence $\ldots \ldots \ldots \ldots \ldots$

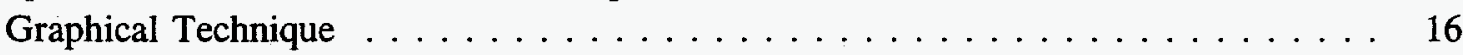

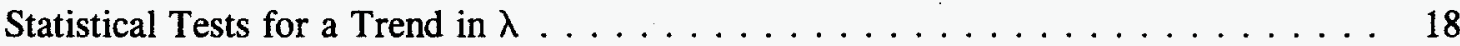

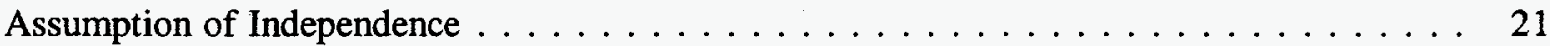

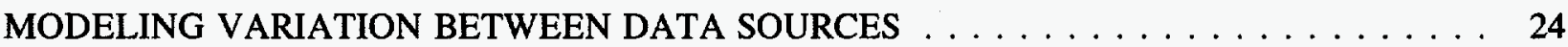

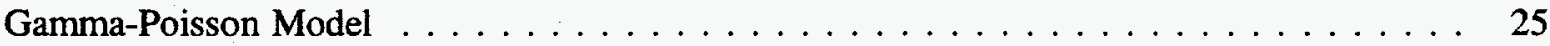

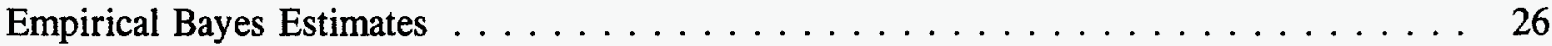

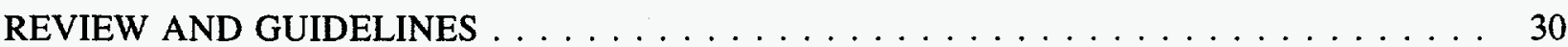

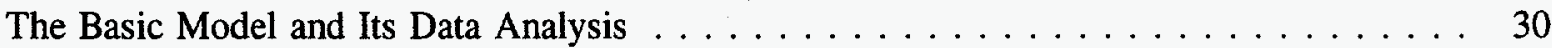

Checking the Assumptions of the Poisson Model $\ldots \ldots \ldots \ldots \ldots \ldots \ldots$

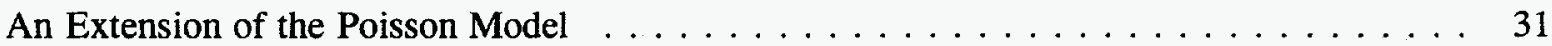

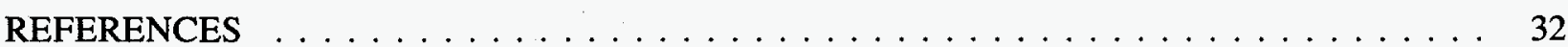

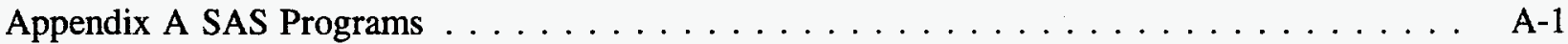

MACRO PCONFINT $\ldots \ldots \ldots \ldots \ldots \ldots \ldots \ldots \ldots \ldots \ldots \ldots \ldots \ldots$

MACRO PBAYES $\ldots \ldots \ldots \ldots \ldots \ldots \ldots \ldots \ldots \ldots \ldots \ldots \ldots \ldots \ldots$

PROGRAM MANN $\ldots \ldots \ldots \ldots \ldots \ldots \ldots \ldots \ldots \ldots \ldots \ldots \ldots \ldots$ 
CONFIDENCE INTERVALS AND BAYES INTERVALS $\ldots \ldots \ldots \ldots \ldots \ldots \ldots$

Confidence Intervals $\ldots \ldots \ldots \ldots \ldots \ldots \ldots \ldots \ldots \ldots \ldots$

Bayes Intervals $\ldots \ldots \ldots \ldots \ldots \ldots \ldots \ldots \ldots \ldots \ldots$

EMPIRICAL BAYES METHOD $\ldots \ldots \ldots \ldots \ldots \ldots \ldots \ldots \ldots \ldots$

Gamma-Poisson Likelihood $\ldots \ldots \ldots \ldots \ldots \ldots \ldots \ldots \ldots$. . . . . . . . . .

Maximum Likelihood Estimation . . . . . . . . . . . . . . . . . B B

Moment Estimation . . . . . . . . . . . . . . . . . . . . B-7

Adjustment for Uncertainty in Maximum Likelihood Estimates . . . . . . . . . . . B-9

REFERENCES $\ldots \ldots \ldots \ldots \ldots \ldots \ldots \ldots \ldots \ldots \ldots \ldots \ldots \ldots \ldots$

\section{FIGURES}

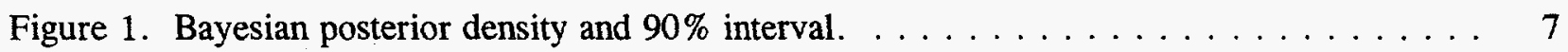

Figure 2. $90 \%$ confidence interval and Bayes $90 \%$ interval. $\ldots \ldots \ldots \ldots \ldots$

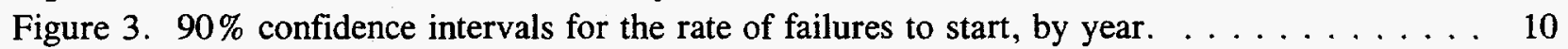

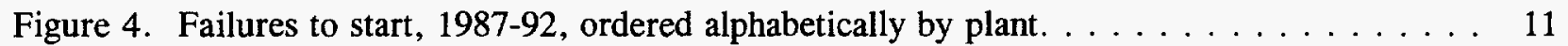

Figure 5. Failures to start, $1987-92$, ordered by rate. $\ldots \ldots \ldots \ldots \ldots \ldots$

Figure 6. Cumulative count of events versus time $\ldots \ldots \ldots \ldots \ldots \ldots \ldots$

Figure 7. Bayes means and $90 \%$ intervals for $\lambda_{i}$, with each plant estimate based on a

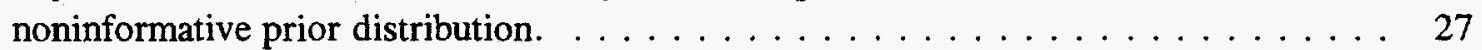

Figure 8. Empirical Bayes means adn $90 \%$ intervals for $\lambda_{i}$, with variance adjustment. . . . . . 28

\section{TABLES}

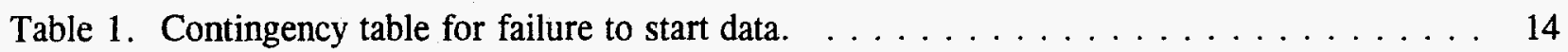

Table 2. Interfailure times in chronological order of failures. $\ldots \ldots \ldots \ldots \ldots$

\section{EXAMPLES}

1. Failures to start, $1987 \ldots \ldots \ldots \ldots \ldots \ldots \ldots \ldots \ldots \ldots \ldots$

2. Failures to start $1987-92 \ldots \ldots \ldots \ldots \ldots \ldots \ldots$

3. Failure to start times, $1987-92 \ldots \ldots \ldots \ldots \ldots \ldots \ldots \ldots$ 


\section{Events in Time: Basic Analysis of Poisson Data}

\section{INTRODUCTION}

This report presents basic statistical methods for analyzing discrete data obtained from counting the numbers of occurrences of events during specified periods of time, and related event-time data. Only basic methods are given here, with more advanced methods deferred to a later report.

The following example illustrates the types of data often encountered in the analysis of event times. For commercial nuclear power plant data, the primary operational concern usually involves the number of failures of some type of component or system occurring during fixed periods of time. The number of failures during any fixed time period, such as a year, is a discrete (integer-valued) random variable. Data obtained this way are called "count-data." Other types of data would involve the exact failuretimes, measured from some fixed point in time, and the times between failures. Although these types of data involve different random variables, we might expect a relationship between the distributions of failure-times, times between failures, and numbers of failures during fixed periods of time. For example, if failure-times are occurring close together, the failure-counts in fixed periods will tend to be high.

This report first treats the basic model, with a single parameter to be estimated, from counts of events in specified disjoint intervals of time. Then it gives ways to check the correctness of the basic model assumptions for data sets. It presents a compound model for situations where different subsets of the data come from sources having different rates of occurrence. Every definition and procedure is illustrated with an example, and references are given for all but the most basic formulas. Appendix A provides SAS (1988) programs to do all of the examples.

a. Mention of specific products and/or manufacturers in this document implies neither endorsement or preference, nor disapproval by the U.S. Government, any of its agencies, or EG\&G Idaho, Inc. of the use of a specific product for any purpose. 


\section{BASIC MODEL}

The analysis methods of this report are illustrated by data from a 1993 draft study. That study considered failures of the high pressure coolant injection (HPCI) system in commercial nuclear power plants of the boiling water reactor design. The HPCI system is a standby system, demanded only on conditions of low water level in the reactor vessel (which automatically initiate unplanned actuations of the system) and periodic surveillance tests. Any failure to operate during either unplanned actuations or surveillance tests must be reported in a Licensee Event Report (LER). These reports were read, and the resulting data formed the basis of the draft study. After peer review, some of the events in the data set were reclassified. Therefore, the example data sets in this report are not real, but they are like real data sets. They are given as illustrations only, and no inferences about the actual systems should be drawn from the numerical conclusions.

The two main aspects of the data are the number of occurrences of the event and the "exposure time," or the amount of time when the system was required to be operational, a fraction of the calendar time.

Example 1. Failures to start, 1987.

In one year of data covering a peer group consisting of six U.S. Boiling water reactors with a high pressure coolant injection system, there were 4 failures to start. The total exposure time for the reactors in this peer group was 4.31 reactor years.

It is instructive to compare this example with a similar example of failure to start on demand in Atwood (1994). In that example, a failure was counted only if it occurred on a demand of the entire system, either an unplanned demand or a full-flow surveillance test. There were 3 such failures in 30 demands in 1987. In the present example, an event occurs whenever a problem is discovered that could result in a failure of the system or some portion of the system. Such a problem might involve, for example, a failure on demand, or a hardware problem, such as a valve that has been inadvertently left closed after maintenance. As stated above, there were 4 such failures in the six plants considered in 1987. 


\section{Assumptions}

The usual assumptions for counts of events in time are:

1. The probability that an event will occur in any specified short exposure time period is approximately proportional to the length of the period. In other words, for an interval of length $\Delta t$ the probability of an occurrence in the interval is approximately $\lambda \times \Delta t$ for some $\lambda>0$.

2. The probability that more than one event will occur in a specified short exposure time period is negligible relative to the probability that exactly one will occur during the period.

3. Occurrences of events in disjoint exposure time periods are statistically independent.

Under the above assumptions the number of occurrences $N$ in exposure time $t$ is a Poisson distributed random variable with mean $\mu=\lambda t$,

$$
P[N=n]=\frac{\mu^{n} e^{-\mu}}{n !} \quad n=0,1,2, \ldots
$$

The parameter $\lambda$ is called the occurrence rate. A detailed discussion of the assumptions and a derivation of the Poisson distribution are given by Meyer (1970, pp. 165-170). Because the count of events during a fixed period is a unitless quantity, the mean number of occurrences $\mu$ is also unitless. However, the rate $\lambda$ depends on the units for measuring time. In other words, the units of $\lambda$ is 1 per unit of time, such as $1 /$ year in the above example.

The Poisson distribution is also often encountered as an approximation for the binomial distribution, which is a model for the number of hits (or successes) in a fixed number of independent trials of a Bernoulli-type experiment with $p$ the probability of a hit on a single trial. When $p$ is small and the number of trials is large, the number of hits is approximately Poisson distributed with mean $p \times$ (number of trials). An important application of the binomial distribution is encountered in the analysis of safety systems that could fail upon demand (see Atwood 1994). Information about the Poisson distribution is given in many statistics books; the facts needed for data analysis are stated in the following subsections.

In the example introduced above, the count-data during the exposure time for 1987 reflect the frequency of events of the type discussed. Assumption (1) implies that the occurrence rate is constant with respect to time, Assumption (2) precludes observing more than one event in very short exposure 
time periods, and Assumption (3) implies that failures to start over disjoint exposure time periods of the year, such as different weeks or months, are independent. Some methods for checking the model assumptions will be discussed in a later section.

\section{Point Estimation}

If $n$ is the observed number of events in a given exposure time period $t$, then the rate of occurrence $\lambda$ is estimated by $\hat{\lambda}=n / t$. It is also possible to combine data from several independent processes, each having the same rate $\lambda$. In particular, suppose the $i$ th Poisson process is observed for time $t_{\mathrm{i}}$, yielding the observed count $n_{\mathrm{i}}$. The total number of event occurrences is $\Sigma_{\mathrm{i}} n_{\mathrm{i}}$ where the sum is taken over all of the processes. The rate $\lambda$ is estimated by $\hat{\lambda}=\Sigma_{\mathrm{i}} n_{\mathrm{i}} / \Sigma_{\mathrm{j}} t_{\mathrm{i}}$. For example, if counts obtained for different years are used to estimate the rate, the estimate is the ratio of the total count to the total exposure time during these years. For simplicity, when the data are combined from several processes, we will denote the total count $\Sigma_{\mathrm{i}} n_{\mathrm{j}}$ by $n$, and the total time $\Sigma_{\mathrm{i}} t_{\mathrm{i}}$ by $t$. The estimate $\hat{\lambda}$ is the maximum likelihood estimate (MLE), as defined in statistics books. In the example, the estimated rate for a plant in 1987 is $\hat{\lambda}=4 / 4.31$ $=0.93$ events per reactor year.

\section{Standard Deviation of Estimator}

The counts per year are random. In other words, if an identical set of plants could be observed during the same years, a different set of counts would likely be observed due to randomness. Similarly, the same plants would probably yield different counts over a different six-year period. Therefore, the estimator is random, and the estimate is simply the observed value for this set of plants during these years. For a Poisson distributed random variable $N$, the mean and variance are the same, $\mu=\sigma^{2}=\lambda t$. Consequently, the standard deviation of $N$ is $[\lambda t]^{1 / 2}$, and the estimated standard error of the estimator $\hat{\lambda}$ is $[\hat{\lambda} t]^{1 / 2} / t=[\hat{\lambda} t]^{1 / 2}$. Thus, the standard error for this example is $[0.93 / 4.31]^{1 / 2}=0.46$ events per reactor year. The estimated standard error expressed in terms of $n$ and $t$ is $n^{1 / 2} / t$.

\section{Confidence Interval for $\lambda$}

Bain and Engelhardt (1992, Section 11.4) give a formula for a $100 \gamma \%$ confidence interval for the mean of a Poisson distribution. It is based on the $\chi^{2}$ distribution, which is tabulated in most statistics books, and which can be found easily by most statistical software packages. As used below, $\chi_{\mathrm{p}}^{2}(\nu)$ is the $p$ th quantile of the $\chi^{2}$ distribution with $\nu$ degrees of freedom. The confidence limits for the mean are easily modified to obtain a $100(1-\alpha) \%$ confidence interval for $\lambda$. The lower limit is 


$$
\lambda_{L}=\frac{\chi_{\alpha / 2}^{2}(2 n)}{2 t}
$$

If $n=0$, then simply set $\lambda_{L}=0$. Similarly, the upper limit is

$$
\lambda_{U}=\frac{\chi_{1-\alpha / 2}^{2}(2 n+2)}{2 t}
$$

The resulting confidence interval is conservative in the sense that the actual confidence level is no smaller than the nominal level of $100(1-\alpha) \%$, but it could be larger. Notice that an upper confidence limit is defined in the case $n=0$. It is reasonable that observing no occurrences of the event would provide some information about how large $\lambda$ might be, but not about how small it might be.

In Example 1, where $n=4$ events were observed in $t=4.31$ reactor years, suppose a $90 \%$ confidence interval is to be found. It has already been determined that the 0.05 th and 0.95 th quantiles are required. Because $n=4$, the $\chi^{2}$ values would be $\chi_{0.05}^{2}(8)=2.73$ and $\chi_{0.95}^{2}(10)=18.31$. The lower limit is $\lambda_{\mathrm{L}}=2.73 /(2 \times 4.31)=0.32$ and the upper limit is $\lambda_{\mathrm{U}}=18.31 /(2 \times 4.31)=2.12$. The resulting $90 \%$ confidence interval, in events per reactor year, is

$(0.32,2.12)$.

Many statistics books mention an approximate confidence interval, based on the approximate normality of $\hat{\lambda}$ In the modern computing environment, there is no reason for ever using that interval in a printed report. The SAS macro pconfint is provided in Appendix A to perform the necessary calculations.

\section{Bayesian Distribution}

If $\lambda$ is to be input to a PRA code such as IRRAS (Russell et al. 1992, 1993), it must be given an uncertainty distribution. The current version of IRRAS does not accept an uncertainty distribution on $\lambda$, but only on $\lambda \tau$, or on $1-e^{-\lambda \tau}$, where $\tau$ is called the "mission time." Uncertainty distributions on $\lambda$ are anticipated in future versions of IRRAS.

We now consider a standard Bayesian approach in which $\lambda$, the rate of occurrence of the Poisson distribution, is assumed to have a gamma prior distribution, denoted by $\operatorname{gamma}(\alpha, \beta)$. The probability density function of this distribution is 


$$
f(\lambda)=\frac{\beta^{\alpha}}{\Gamma(\alpha)} \lambda^{\alpha-1} \exp (-\beta \lambda)
$$

if $\lambda>0$, and zero otherwise. The mean and variance of the $\operatorname{gamma}(\alpha, \beta)$ distribution are, respectively, $\alpha / \beta$ and $\alpha / \beta^{2}$. The parameter $\alpha$ is called a shape parameter, and $\beta$ is called a scale parameter. The reciprocal of $\beta$ (a parameterization preferred by some statisticians) is also referred to as a scale parameter. The shape parameter $\alpha$ is unitless, and in the application to failure rates, the scale parameter $\beta$ has units of time so that $\beta \lambda$ is unitless.

Denote by $n$ the total of the observed event counts, which are assumed Poisson with the same rate $\lambda$, and denote by $t$ the total exposure time. It is shown in Appendix B that if $\lambda$ has prior distribution $\operatorname{gamma}(\alpha, \beta)$, and the event counts are independent, the posterior distribution of $\lambda$ is $\operatorname{gamma}(\alpha+n, \beta$ $+t$ ). It is helpful to think of the parameters of the posterior distribution as being updated by the data from those of the prior distribution. Specifically, formulas for updating the parameters are $\alpha_{\text {post }}=\alpha_{\text {prior }}$ $+n$ and $\beta_{\text {post }}=\beta_{\text {prior }}+t$.

A special type of prior distribution is obtained if the shape parameter is taken to be $\alpha=1 / 2$ and the parameter $\beta$ is taken to be zero. This gives a function that is proportional to $\lambda^{-1 / 2}$. Although this function is interpreted as a density function, it is called "improper" because its integral, with limits 0 to $\infty$, is infinite. This function of $\lambda$ is also known as the Jeffreys "noninformative prior" (Box and Tiao 1973). The Bayes posterior distribution for $\lambda$ in this case is gamma $(n+1 / 2, t)$. The SAS macro pbayes is provided in Appendix A to perform the necessary calculations.

For comparison with confidence intervals, it is convenient to express the gamma $(n+1 / 2, t)$ posterior distribution in terms of a $\chi^{2}$ distribution. A random variable $Y$ has a $\chi^{2}$ distribution with $\nu$ degrees of freedom if it is $\operatorname{gamma}(\nu / 2,1 / 2)$. It is helpful to note that if $X$ is $\operatorname{gamma}(\alpha, \beta)$, then $2 \beta X$ is $\chi^{2}$ with $\nu$ $=2 \alpha$ degrees of freedom. With this relationship, the above Bayesian analyses can be conveniently stated in terms of the $\chi^{2}$ distribution. Specifically, because the noninformative prior leads to a gamma $(n+1 / 2$, $t$ ) posterior distribution for $\lambda$, the posterior distribution of $2 t \lambda$ is the $\chi^{2}$ distribution with $\nu=2 n+1$ degrees of freedom. For example, if a Bayesian $90 \%$ interval is required, the interval $\left(\lambda_{1}, \lambda_{2}\right)$ with limits $\lambda_{1}=\chi_{0.05}^{2}(2 n+1) /(2 t)$ and $\lambda_{2}=\chi_{0.95}^{2}(2 n+1) /(2 t)$ provides such an interval. We note the similarity between the Bayes limits and the confidence limits stated earlier. The manner of computing the degrees of freedom is slightly different for the two types of intervals. As a result, the intervals are numerically similar, although the interpretation is somewhat different. Thus, both types of intervals should be numerically close, with the Bayes interval slightly shorter at each end. The mean of the Bayes posterior distribution for the rate $\lambda$ is $[n+1 / 2] / t=\hat{\lambda}+1 /(2 t)$. 
In Example 1, the posterior distribution of $2 t \lambda$ is $\chi^{2}$ with $\nu=2 \times 4+1=9$ degrees of freedom. This results in a Bayes $90 \%$ interval estimate with lower limit $\lambda_{1}=3.325 /(2 \times 4.31)=0.39$ events per reactor year and upper limit $\lambda_{2}=16.919 /(2 \times 4.31)=1.96$ events per reactor year. In this example, the mean of $\lambda$ is $0.93+1 /(2 \times 4.31)=1.05$ events per reactor year, and the shape parameter is $4+$ $1 / 2=4.5$. The posterior distribution and interval for $\lambda$ are illustrated in Figure 1 using data from Example 1.

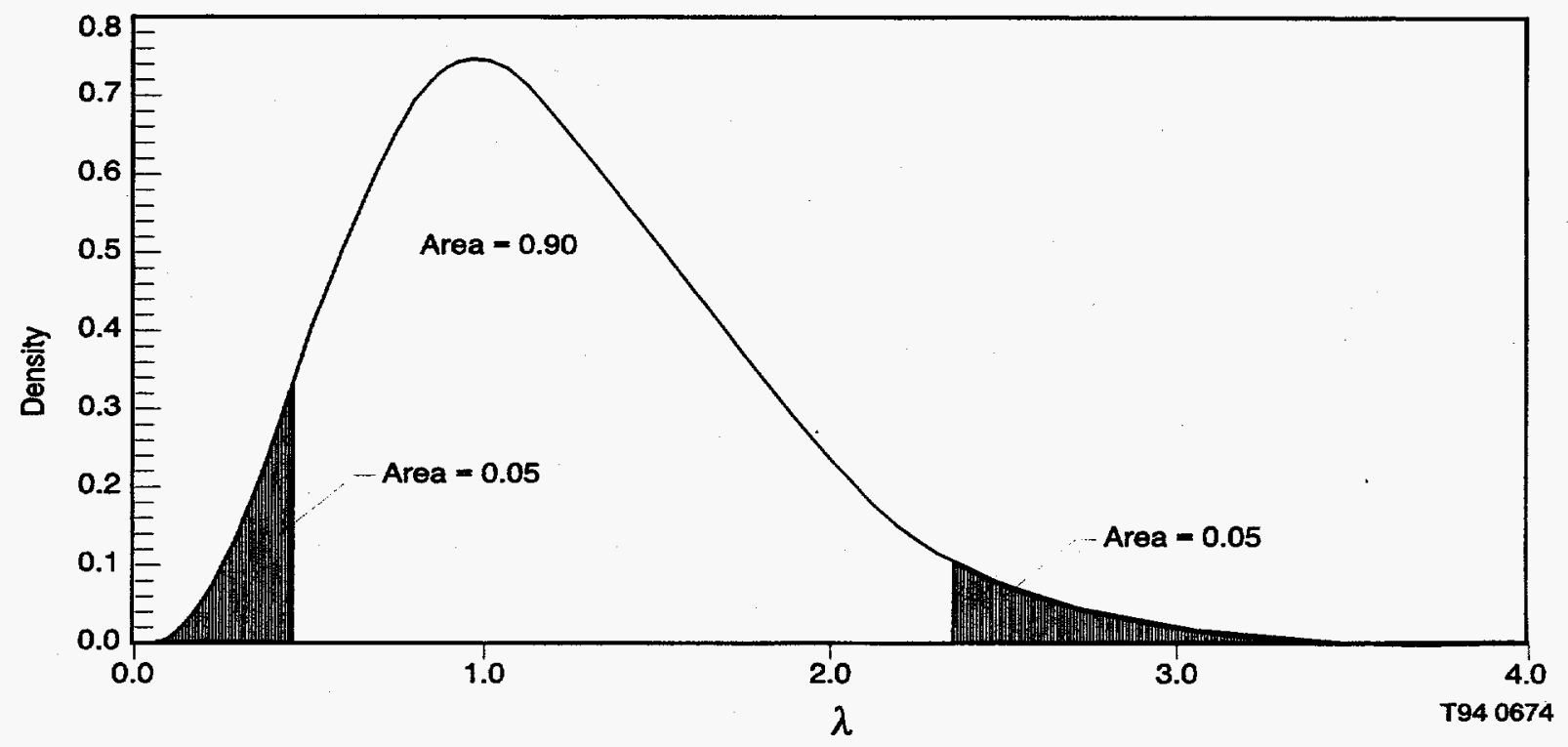

Figure 1. Bayesian posterior density and $90 \%$ interval.

Figure 2 provides a comparison between $90 \%$ confidence limits and the corresponding Bayes limits for $\lambda$. 


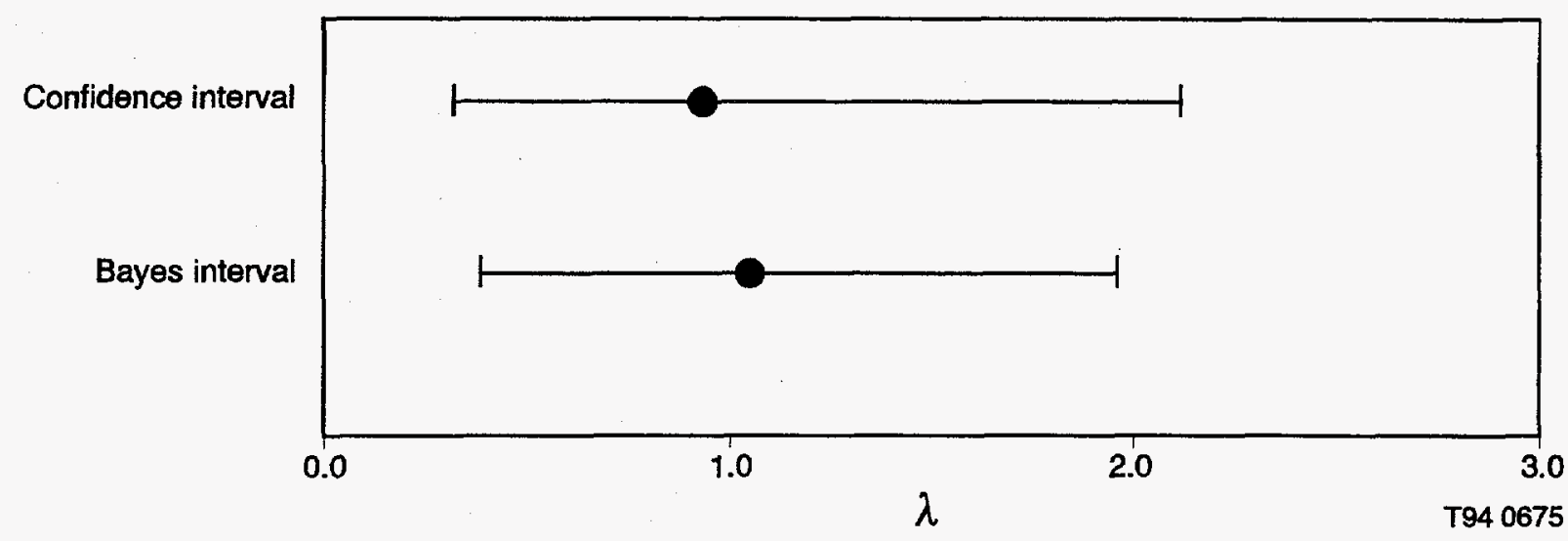

Figure 2. $90 \%$ confidence interval and Bayes $90 \%$ interval. 


\section{CHECKING THE MODEL ASSUMPTIONS}

An important part of any data analysis involves checking the assumptions of the model. Several possible violations of the basic Poisson assumptions are considered here. For each case, one or more graphical techniques are given, to help the analyst understand what the data set shows. Then, formal statistical tools are presented, which quantify the strength of any evidence against the assumption being considered.

\section{Assumption of Same $\lambda$ in Different Subsets}

Recall that Assumption 1 implied that there is one rate $\lambda$ for the entire period of observation. The correctness of such an assumption can be investigated by analyzing subsets of the data, obtained separately over different intervals, and comparing the $\lambda$ 's for the various subsets. For a numerical example, we consider system failure counts previously mentioned.

An obvious question concerns the possibility of differences in rate for different years of operation. Other questions of interest would deal with differences between plant peer groups or between individual plants. It should be noted that the finer the division of the groups, the more sparse the data becomes within the groups. Once the data are grouped and the $\lambda$ 's are computed, the procedures described below can be carried out to help detect any real differences.

The methods will be illustrated in terms of differences in years 1987 through 1992 for the plants mentioned above. The data given in Example 2 include the data for 1987 used in Example 1.

Example 2. Failures to start, 1987-92.

\begin{tabular}{|cccc|}
\hline Years & Number of events & Reactor years & Estimate \\
1987 & 4 & 4.31 & 0.93 \\
1988 & 5 & 4.06 & 1.23 \\
1989 & 3 & 4.02 & 0.75 \\
1990 & 5 & 5.07 & 0.99 \\
1991 & 5 & 5.23 & 0.96 \\
1992 & 4 & 5.02 & 0.80 \\
Total & 26 & 27.69 & 0.94 \\
\hline
\end{tabular}


First, some graphical techniques are given, to help the analyst understand what the data set shows. Then, formal statistical procedures are discussed that help quantify the strength of the evidence for trends or patterns seen in the graphical investigation.

\section{Graphical Techniques}

To explore the relations between subsets of the data, identify the subsets on one axis. Then, for each of these subsets of the data, plot an estimate of $\lambda$ and a confidence interval for $\lambda$ against the other axis. Patterns such as trends, outliers, or large scatter are then made visible.

In Example 1 the subsets are depicted as years. The data set from each year is to be analyzed separately, and the graph is to show an estimate and a confidence interval for each year, plotted side by side. As shown in Figure 3, we place the years on the vertical axis and plot the estimates and confidence intervals against the horizontal axis. The intervals were computed by the SAS macro, called pconfint, which is given in Appendix A.

For each year, a point estimate and a $90 \%$ confidence interval for $\lambda$ have been found. The calculations were performed just as in the example for a single year. For this report, the plot was produced with a graphics software package, although a hand-drawn sketch would be adequate to show the results. The results for 1989 appear a bit lower than the others. The exposure time for this year was the smallest of

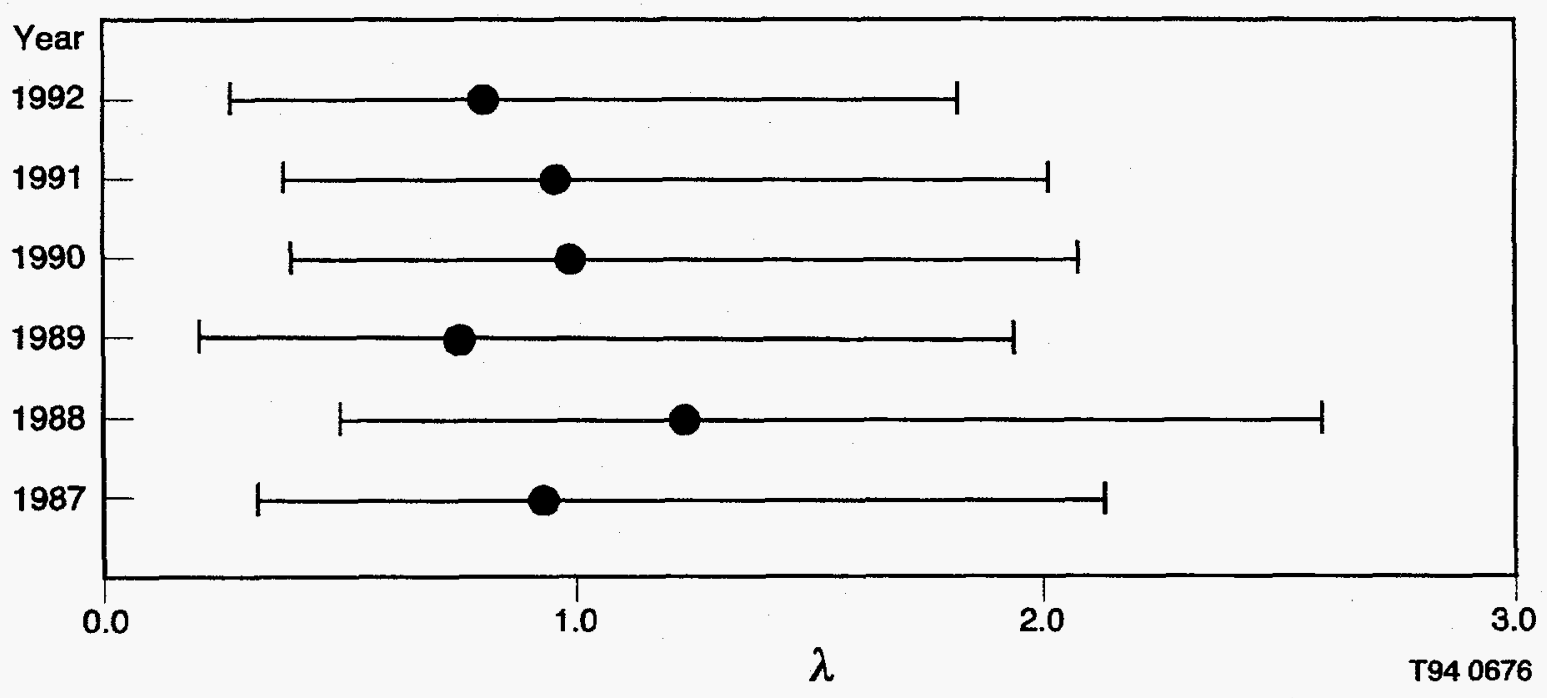

Figure 3. $90 \%$ confidence intervals for the rate of failures to start, by year. 
the six years, and the number of events observed this year was also the smallest. There is considerable overlap among the confidence intervals, and consequently the difference could have easily resulted from random variation. It appears acceptable to pool the data from the six years.

Sometimes a variation of this plot is useful, in which the confidence interval for the pooled data is shown. To illustrate this method, we consider failure data from 23 power plants with boiling water reactors and HPCI systems during the years 1987 through 1992. As with the earlier examples, the data come from a draft study which has since been changed. Therefore, the names of the plants have been changed to generic names, to prevent anyone from drawing erroneous conclusions about particular plants.

The data sources in Figure 3 are plotted in a natural order, from 1987 to 1982, while the data sources in Figure 4 are plotted in a completely arbitrary order, by alphabetic code names.

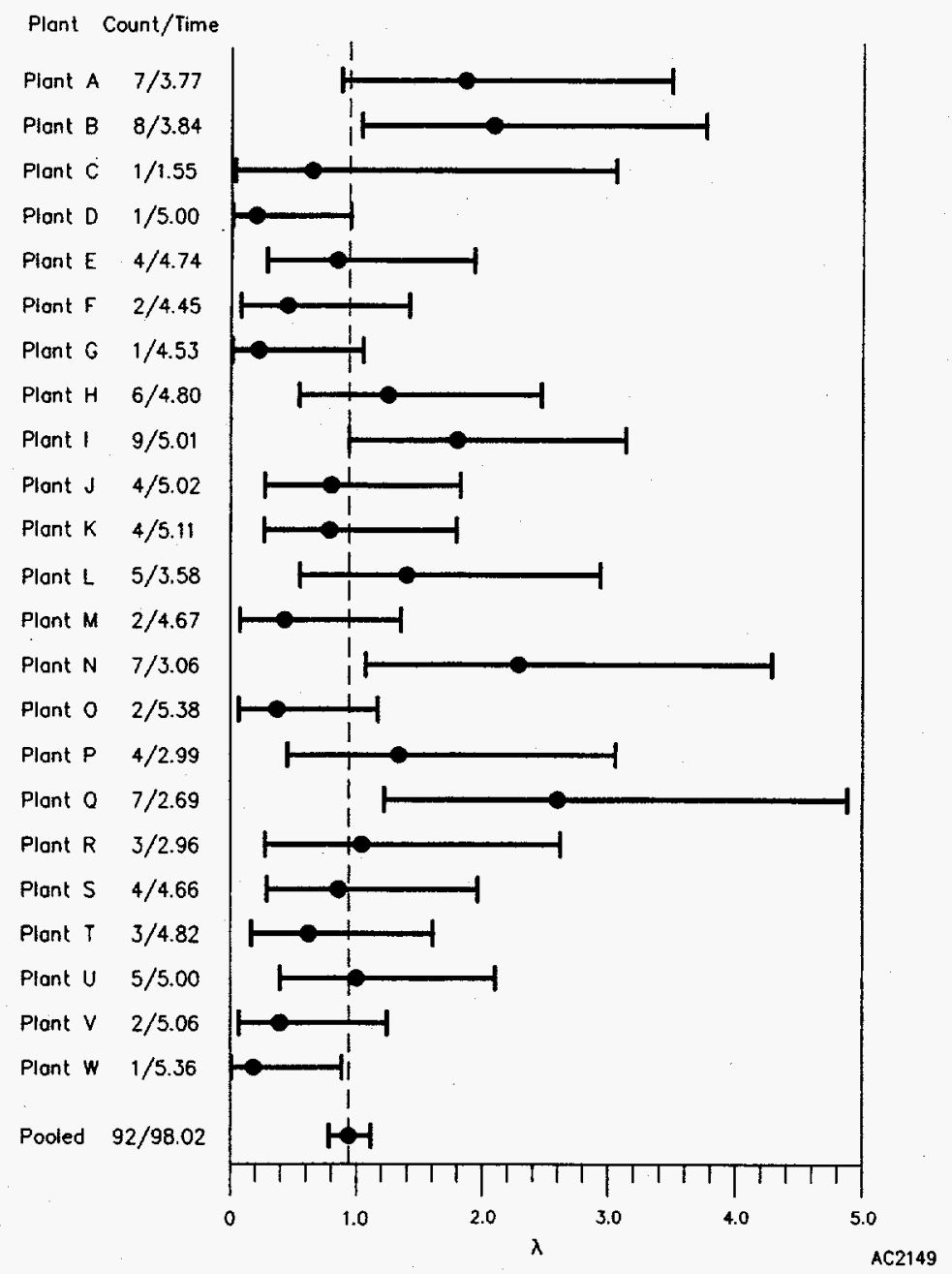

Figure 4. Failures to start, 1987-92, ordered alphabetically by plant. 
When the data sources are ordered arbitrarily, it can be helpful to arrange them in some more meaningful order. For example, the plants in Figure 4 could be arranged in order of plant age, if the analyst wants to investigate whether plant age is related to system reliability. Alternatively, the plants could be ordered so that those with similar design characteristics are grouped together. One useful ordering, which does not require any information about the data sources other than the failure counts and the exposure times, is from worst to best. Figure 5 is such a graph.

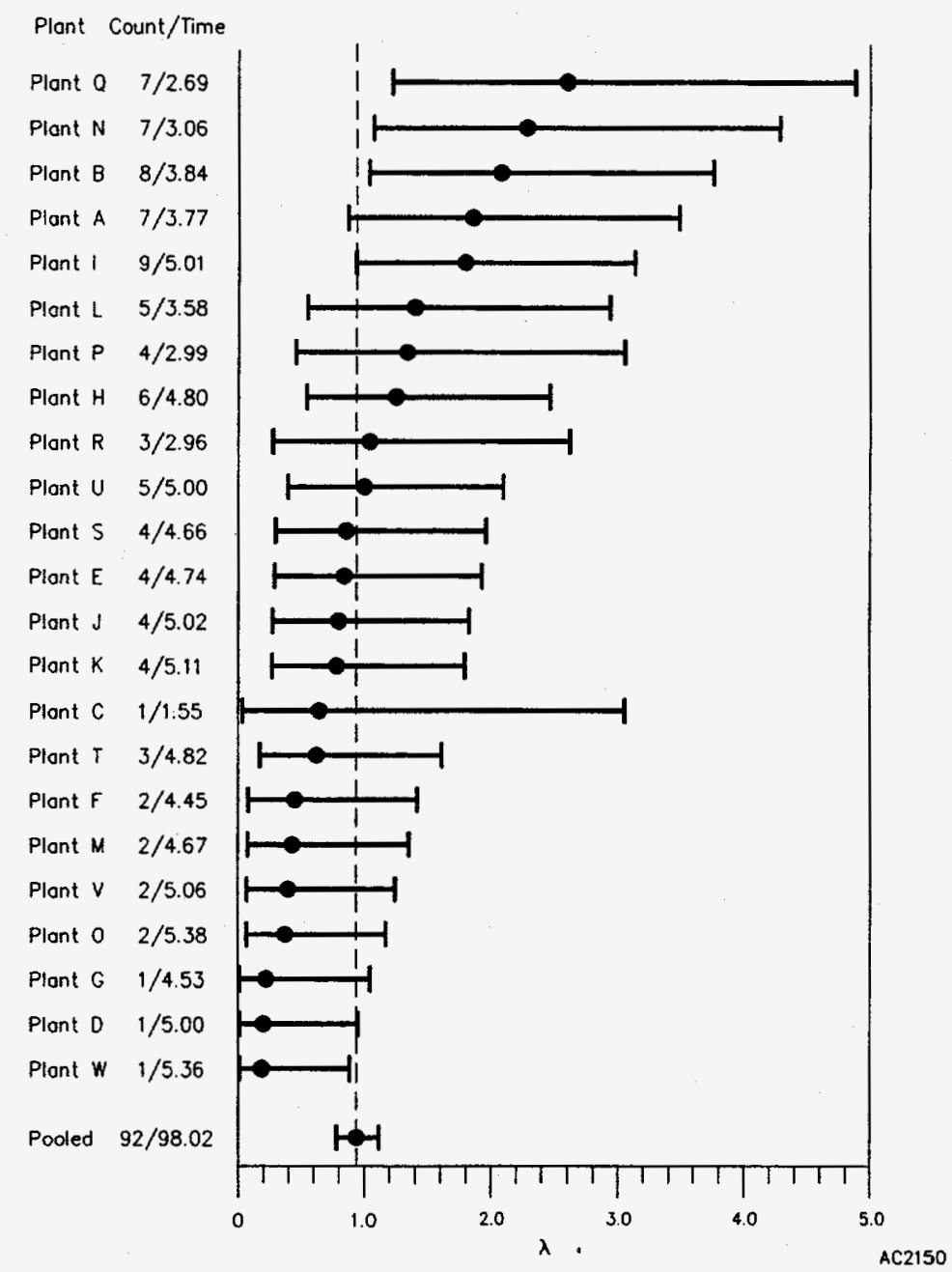

Figure 5. Failures to start, 1987-92, ordered by rate.

The rows of Figure 4 have simply been rearranged, so that $\hat{\lambda}$ is decreasing from top to bottom. This plot makes it easy to spot which plants performed worst in this data set, and to answer questions such as whether the worst plant is much worse than the second worst. Cleveland (1985, Chap. 3.3) discusses this and other ways of ordering data.

Figures 4 and 5 also include a dotted reference line which is drawn through the point estimate based on the pooled data. If there are only a few data subsets to be compared, this enhancement may not be 
necessary. However, if there are many subsets, the picture becomes more confusing, and it becomes more difficult to focus without the reference line. Although in Figures 4 and 5 there is considerable variation in the estimates of $\lambda$, there is also some overlap among all of the confidence intervals. This suggests that the variations may well be due to random causes.

A graph like this should not be used to draw naive conclusions without also using a formal statistical test. For example, if many confidence intervals are plotted, all based on data from a data source with the same $\lambda$, a few will be far from the others because of randomness alone. Thus, an outlying interval does not prove that the $\lambda$ 's are unequal. However, if there are few intervals, intervals that just barely overlap can give strong evidence for a difference in the $\lambda$ 's. To quantify the strength of the evidence based on graphical methods, a formal statistical procedure is given in the next subsection. The graph gives an indication of what the test might show, and helps in the interpretation of the formal statistical quantification. If the statistical test turns out to find a statistically significant difference between years, we naturally would then ask what kind of difference is present. Figure 3 shows that there is not a steadily increasing or decreasing $\lambda$. Instead, any difference between years seems to be the result of randomness. Unusually long intervals, such as that seen in Figure 3 for 1988 or in Figure 4 for Plant $\mathrm{Q}$, are generally associated with a smaller exposure time. The picture provides insight even though it does not give a quantitative statistical test.

\section{Statistical Tests}

Simple Contingency Tables $(1 \times c)$. A simple way to test whether the rate is the same for different data sources is the use a "chi-square" test. A discussion of this subject is given by Bain and Engelhardt (1992, Chapter 13), and many other general statistics texts also have a chapter on this topic. Chi-square tests will be explained here and illustrated by an example. In general, for a two-way table, attributes of the events are associated with $r$ rows and $c$ columns, and the numbers in the table are counts. For the data of Example 2, the only attribute is the year, and the count, $n_{\mathrm{j}}$, is the number of observed events during the reactor year. Thus, in this case, there are $r=1$ rows and $c=6$ columns. This corresponds to a $1 \times c$ table. In this situation, it is more natural to think of the columns as "cells" that contain the counts. The general method is based on comparing the observed counts $n_{\mathrm{j}}$ and the corresponding estimates of expected counts $e_{\mathrm{j}}$ for each cell. Assuming the hypothesis of a constant rate $\lambda$, an estimate of the expected count for the $j$ th cell is simply $e_{j}=\hat{\lambda}_{\mathrm{j}}$ where $\hat{\lambda}=\Sigma_{\mathrm{i}} n_{\mathrm{i}} / \Sigma_{\mathrm{i}} t_{\mathrm{j}}$. Table 1 is a $1 \times 6$ table with the observed counts taken from Example 2. It is convenient to also include the exposure times and expected counts. The grand totals for the observed counts and the exposure times are given in the right margin of the table. These can be used to compute a pooled estimate $\hat{\lambda}=26 / 27.69=0.94$ per reactor 
year. The grand total of the expected counts should agree with the grand total of the observed counts, but they can differ slightly due to round off error.

Table 1. Contingency table for failure to start data.

\begin{tabular}{llllllll}
\hline & 1987 & 1988 & 1989 & 1990 & 1991 & 1992 & Total \\
\cline { 2 - 8 } Observed count & 4 & 5 & 3 & 5 & 5 & 4 & 26 \\
Exposure time & 4.31 & 4.06 & 4.02 & 5.07 & 5.23 & 5.02 & 27.69 \\
\hline Expected count & 4.05 & 3.82 & 3.78 & 4.77 & 4.92 & 4.72 & \\
\hline
\end{tabular}

In the present application, let $H_{0}$ denote the hypothesis that $\lambda$ is the same in all six years. The method used is to see what kind of data would be expected when $\lambda$ really is the same, and then to see how much the observed counts differ from the expected. If the difference is small, the counts are consistent with the hypothesis $H_{0}$ that the rate is constant. If, instead, the difference is large, the counts show strong evidence against $H_{0}$.

If $H_{0}$ is true, that is, if $\lambda$ is really the same for all the years, then for 1987 we would estimate $0.94 \times 4.31=4.05$ events on average; for $1988,0.94 \times 4.06=3.82$ events, and so forth. The test for equality of rates that is considered here is based on an approximate $\chi^{2}$ statistic,

$X^{2}=\Sigma_{\mathrm{j}}\left(n_{\mathrm{j}}-e_{\mathrm{j}}\right)^{2} / e_{\mathrm{j}}$

When $H_{0}$ is true and the total count is large, the distribution of $X^{2}$ has a distribution that is approximately $\chi^{2}$ with $v=c-1$ degrees of freedom. If $X^{2}$ is large, compared to the $\chi^{2}$ distribution, there is strong evidence that $H_{0}$ is false; the larger the $X^{2}$ value, the stronger the evidence.

Suppose, for example with six cells, $X^{2}$ were 11.6. A table of the $\chi^{2}$ distribution shows that 11.1 is the 0.95 th quantile of the $\chi^{2}$ distribution with 5 degrees of freedom, and 12.8 is the 0.975 th quantile. After comparing $X^{2}$ to these values, we would conclude that the evidence is strong against $H_{0}$, but not overwhelming. Quantitatively, we would reject $H_{0}$ at the $5 \%$ significance level, but not at the $2.5 \%$ significance level. This is sometimes phrased as "the p-value is between 0.05 and 0.025 ." If $X^{2}$ were 5.1 , it lies between the 0.50 th and the 0.70 th quantiles of the $\chi^{2}$ distribution, and therefore is in the range of values that would be expected under $H_{0}$. We would say the observed counts are consistent with the hypothesis $H_{0}$, or $H_{0}$ cannot be rejected, or the evidence against $H_{0}$ is weak. We would not conclude that $H_{0}$ is true, because it probably is not exactly true to the tenth decimal place, but the conclusion would be that it cannot be rejected by the data. 
The above considerations are valid if the $e$ 's are "large." If the $e$ 's are small, the $X^{2}$ distribution does not provide a good approximation. Thus, the user must ask how large a count is necessary for the $\chi^{2}$ approximation to be adequate. An overly conservative rule is that all the expected cell-counts, $e_{j}$, should be 5.0 or larger. Despite its conservatism, this rule is still widely used, and cited in the statistical literature.

A discussion of $\chi^{2}$ goodness-of-fit statistics by D'Agostino and Stephens (1986, p.71) is applicable here. Citing the work of Roscoe and Byars (1971), the following recommendations are made: (1) "With equiprobable cells, the average expected frequency should be at least 1 when testing fit at the $\alpha=0.05$ level." In other words, they recommend use of the $\chi^{2}$ approximation at the $5 \%$ level when $n / c \geq 1$. At the $1 \%$ level, the $\chi^{2}$ approximation is recommended if $n / c \geq 2$. They state further: (2) "When cells are not approximately equiprobable, the average expected frequencies in (1) should be doubled." Thus, they recommend that at the $5 \%$ level, $n / c \geq 2$, and at the $1 \%$ level, $n / c \geq 4$.

Note that in rules (1) and (2) above, the recommendation is based on the average rather than the minimum expected cell-count. As noted in another study by Koehler and Larntz (1980), any rule such as (2) may be defeated by a sufficiently skewed assignment of cell probabilities.

Roscoe and Byars also recommend when $c=2$ that the $\chi^{2}$ test should be replaced by the test based on the exact binomial distribution. As noted by Cox and Lewis $\left(1978\right.$, p. 224), if $c=2$, and $N_{1}$ and $N_{2}$ are the random cell-counts, then conditional on $N_{1}+N_{2}=n, N_{2}$ is binomial with parameters $n$ and $p$ $=\lambda_{2} t_{2} /\left(\lambda_{1} t_{1}+\lambda_{2} t_{2}\right)$. If the rate is the same $\left(\lambda_{1}=\lambda_{2}=\lambda\right)$, the parameter $p$ is free of the unknown value $\lambda$, and a test of the null hypothesis $\mathrm{H}_{0}: p=t_{2} /\left(t_{1}+t_{2}\right)$ based on $N_{2}$ provides a test of constant rate. Exact binomial tests are discussed by Bain and Engelhardt (1992, p.405).

For a test at either the $1 \%$ or $5 \%$ level, Table 1 is all right as it stands according to the RoscoeByars guidelines because $n / c=26 / 6 \geq 4$. The $\chi^{2}$ statistic, as computed by SAS, is $X^{2}=0.65$ with $\nu$ $=6-1=5$ degrees of freedom. As noted above, the 0.95 th quantile of the $\chi^{2}$ distribution with $\nu=$ 5 degrees of freedom is 11.1 , and thus, at the $5 \%$ level the test does not reject the null hypothesis of constant rate. We conclude that there is no evidence of a difference between years, just as we suspected after looking at Figure 3. As noted above, SAS can be programmed to perform chi-square tests (see the macro pconfint in Appendix A), but in the case of a 1 by $c$ table (with small $c$ ), the statistic is easily computed with the aid of a hand-held calculator.

With guidelines based on either minimum or average expected cell-counts, if the expected cellcounts are so small that the $\chi^{2}$ approximation is not recommended, the analyst can pool data in some 
adjacent cells, thereby increasing the expected cell-counts. For example, suppose it is desired to use the conservative rule in which no expected cell-count is less than 5.0. This could be accomplished by pooling 1987 and 1988 into one cell, 1989 and 1990 into a second cell, and 1991 and 1992 into a third cell. For the data of Table 1, with the data pooled in this manner, the degrees of freedom would be $\nu=3-1=2$.

Generally speaking, a $\chi^{2}$ test based on a larger number of cells will have better power for detecting when rates are not equal, but this also makes it more difficult to satisfy guidelines on expected cell-counts for the $\chi^{2}$ approximation. Thus, it is sometimes necessary to make a compromise between expected cellcounts and the number of cells.

Other options, involving the exact distribution of $\chi^{2}$, are also possible. An approximation to the exact distribution of the $\chi^{2}$ statistic, with $e_{\mathrm{j}}$ small for some or all cells, is given by Atwood (1981). Also, a program called HOMOG, for testing the homogeneity of Poisson data sources, is discussed by Atwood (1982). The original version of this program, which was written in CDC Fortran for use on a Cyber mainframe computer, has been rewritten to run on a PC using the MS-DOS operating system, although no new documentation is currently available.

This $\chi^{2}$ method does not use any ordering of the data subsets. In the example, the ordering is natural, the chronological order from 1987 through 1992. Other applications might not have an obvious natural ordering of the subsets. For example, another potential application would be to test whether the different plants in the peer group have the same value of $\lambda$. In this example, outputs of the data would likely be alphabetical by plant identifier, in which case the order is arbitrary. The analysis method is exactly the same in the two cases, ignoring the natural order that may be present. When there is a meaningful order to the data subsets, it may be useful to perform additional analyses. The analysis given above is valid, but an additional possible analysis, making use of time order, is considered next.

\section{Assumption of Constant $\lambda$ for Events in Sequence}

The following tools are useful if the individual events can be arranged in a single sequence, for example if the dates are known.

\section{Graphical Technique}

A very basic plot is a cumulative plot of the number of events against time. Other types of plots are possible, but they will not be considered in this report on basic methods. 
For the purpose of illustration, the cumulative numbers of events during 1987-1992, given in Example 3, are plotted against time. It should be noted that in this example the time axis represents "calendar" years and not reactor years. It is generally somewhat more difficult from LER data to determine the occurrence times corresponding only to reactor years. Thus, calendar years are being used here to provide a simple example. January 1987 was taken to be calendar year 0; thus, all reporting

Example 3. Failure to start times, 1987-92.

$0.12,0.15,0.28,0.49,1.03,1.28,1.38,1.57,1.84,2.29,2.44,2.79,3.13,3.19,3.30,3.68$, $3.80,4.11,4.70,4.88,4.89,4.95,5.01,5.10,5.15,5.20$

times are inclusive between 0 and 6 . For example, the first and second failures occurred on February 14 and 23 of 1987, so that the first two failure times in Example 3 are, respectively, 45/365 $=0.12$ and $54 / 365=0.15$ calendar years. It should also be noted that a rather lengthy gap exists between the last reported failure time and the end of the study. It was not possible to determine from the data base whether some of the data from the last part of the study is missing, or whether no failures actually occurred during this period.

The LERs were arranged in chronological order, and the cumulative count of FTS events was plotted against the reporting times in Figure 6.

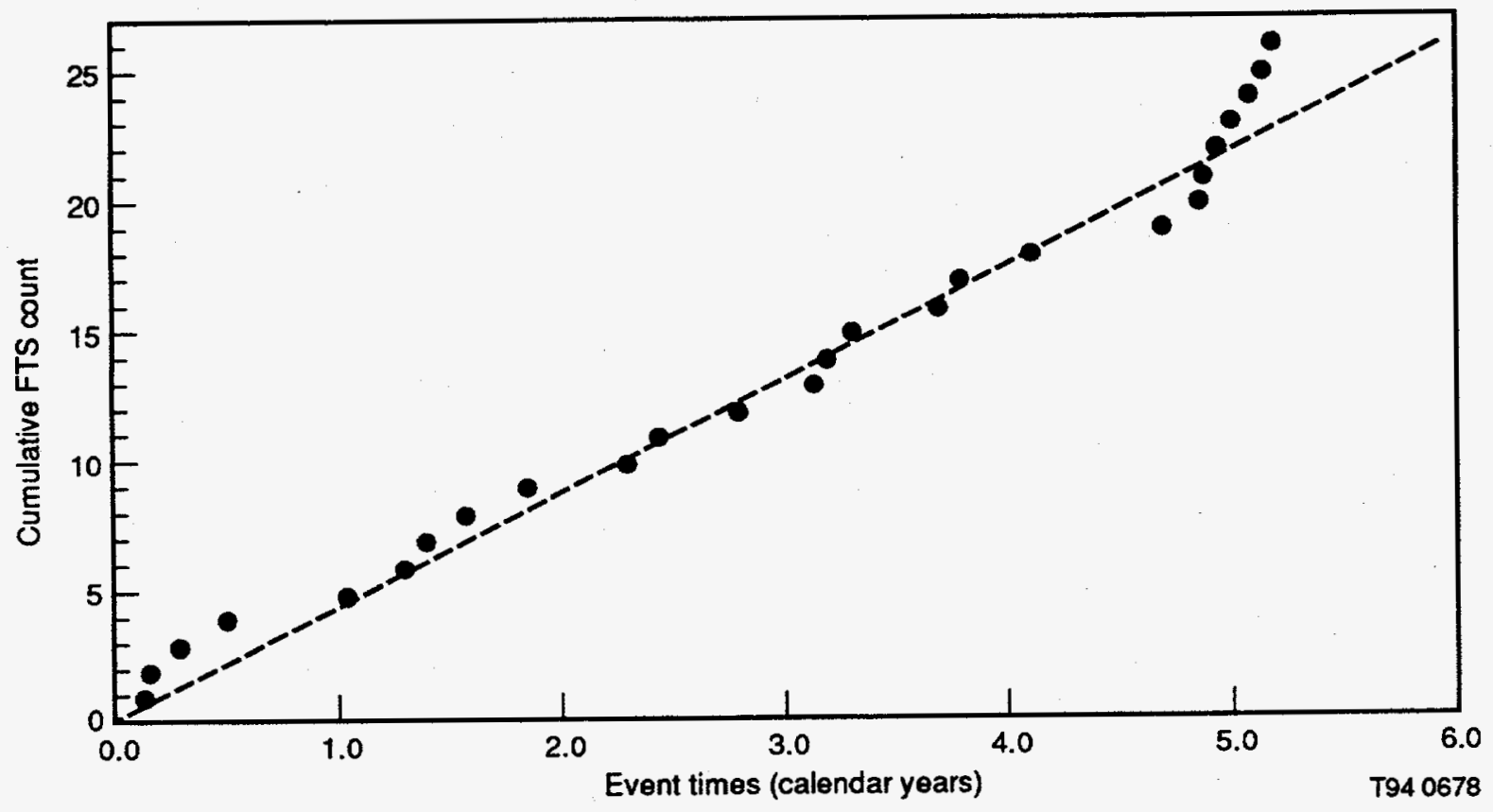

Figure 6. Cumulative count of events versus time. 
The dotted line shown in Figure 6 is the graph of the estimated mean, which is $\hat{\mu}=\hat{\lambda} t$ plotted as a function of calendar time, $t$. The estimated rate is $\hat{\lambda}=26 / 6$ failures per calendar year, and thus the dotted line connects the origin $(0,0)$ with the point $(6,26)$.

Such a plot can be used as an exploratory technique. The two main objectives of the plot are (1) to check model assumptions, such as constant rate, and (2) to identify outliers or mixtures of data.

In this example, the points toward the center of the graph (roughly from 1.0 to 5.0 ) tend to bounce around the dotted line, as would be expected with purely random variation. However, there are a few points, both at the start and also at the end of the plot, which suggest that something other than just random variation could possibly be present. Specifically, the last 7 points tend to rise more rapidly than would be expected with the assumption of a common rate for all six years. Also, the first 4 points tend to rise faster than those in the center of the plot, and they are all above the dotted line.

Such behavior tends to occur when the data is a mixture from two or more data sources with differing rates. A characteristic of plots involving mixed data is that different lines appear to fit different portions of the graph. A common example of this occurs with components such that some have manufacturing defects, and the rest do not. The defective ones tend to fail earlier, and at a higher rate than the ones without defects. This typically results in a subset of points at the beginning of the plot which tend to cluster about a line with a higher than average slope.

It should be noted that mixtures are only one possible explanation for the type of clustering seen in Figure 6. An underlying model with a nonconstant rate would be another possible explanation. Also, in a few cases such apparent patterns will occur from randomness alone. For this reason, the analyst is cautioned against attempting to interpret every small blip in a plot.

An explanation of the apparently differing rates both at the beginning and end for the plot of Figure 6 is not known at this time, but one could possibly be found through an engineering study. A formal test for clustering, would also be helpful in settling this question. Such a test is an advanced topic which is beyond the scope of this report. Our main purpose here is to discuss basic statistical tools for helping to detect trends and quantify their significance.

\section{Statistical Tests for a Trend in $\lambda$}

The Lap/ace Test. A formal statistical test is sometimes needed to provide decisive evidence, particularly in the case of a borderline visual impression. In the example, there are 26 occurrences of 
events during a six-year period. In general, consider a time interval $[0, L]$, and suppose during this period $n$ events occur at successive times $T_{1}, T_{2}, \ldots, T_{n}$. Note that the number of occurrences, $n$, is random when the plants are observed for a fixed length of time $L$. Consider the null hypothesis $H_{0}$ that the rate is constant.

Potentially, there are many tests of $H_{0}$ that could utilize the event times. Against the alternate hypothesis that the events tend to occur more at one end of the interval (i.e., $\lambda$ is either an increasing or a decreasing function of time), a test that is often used is based on the mean of the failure times, $\bar{T}=$ $\Sigma_{\mathrm{i}} T_{\mathrm{i}} / n$. When $H_{0}$ is true, $\bar{T}$ has expected value $L / 2$ and variance $L^{2} /(12 n)$, and the resulting test statistic is

$$
U=\frac{\bar{T}-\frac{L}{2}}{L \sqrt{\frac{1}{12 n}}}
$$

An intuitive explanation of this test is that if events are tending to occur more frequently at one end of the interval, then the mean of the occurrence times will be shifted toward that end. For example, if the rate is increasing, then more of the events will occur near the right endpoint of the interval. Consequently, more of the times will fall above $L / 2$, which is the midpoint of the interval. Thus, positive values of the difference $\bar{T}-L / 2$ indicate an increasing trend, and negative values indicate a decreasing trend. Of course, when the rate is constant, we expect the difference to be near zero.

This test, generally known as the "Laplace" test, is discussed by Cox and Lewis (1978, p. 47). The Laplace test is known to be good for detecting a wide variety of monotonic increasing or decreasing alternatives, and consequently it is recommended as a general tool for testing against such alternatives.

The statistic $U$ defined above is approximately standard normal for $n \geq 3$. A test of $H_{0}$ at significance level 0.05 versus an increasing alternative would reject if $U \geq 1.645$, and a 0.05 level test versus a decreasing alternative would reject if $U \leq-1.645$. Of course, \pm 1.645 are the 0.95 th and 0.05 th quantiles, respectively, of the standard normal distribution. For the data of Example 3 , there are 26 occurrence times with a mean of 2.95 , yielding a value $U=-0.15$ for the test statistic. This value of $U$ is very close to zero, and does not provide any evidence at all of a nonconstant rate of occurrence.

The Mann Test. An interesting property holds for the times between occurrences (interarrival times) of Poisson events with constant rate $\lambda$. Specifically, the interarrival times are independent random variables each exponentially distributed with mean $1 / \lambda$. Another test for trend, which is based on this 
property, is known as the "Mann" test. This test, which is discussed by Ascher and Feingold (1984, page 80), is based on the tendency for interarrival times to become shorter if the rate of occurrence is increasing. The test is performed by counting the "reverse arrangements" among the chronologically ordered interarrival times. Specifically, if we denote the $i$ th interarrival time by $X_{\mathrm{i}}=T_{\mathrm{i}}-T_{\mathrm{i}-1}$ if $i>1$ and with $X_{1}=T_{1}$, then a reverse arrangement occurs whenever $X_{\mathrm{i}}<X_{\mathrm{j}}$ for $i<j$. The Mann test is based on the statistic $R=$ total number of reverse arrangements. In other words, $R$ is computed by comparing every interarrival time with every later interarrival time. Fewer reverse arrangements would be expected if interarrival times are tending to get shorter, and thus a small value of $R$ would indicate an increasing rate of occurrence. For $n$ occurrence times, there are $n(n-1) / 2$ comparisons. It should be noted that when the data are obtained by observing $n$ occurrences during a fixed period of time, the exact value of the interarrival time between the $n$th to the $(n+1)$ st occurrence will not be known. It might be possible to increase the power of the test by using the fact that no events occurred between the $n$th occurrence and the end of the observation period, but for simplicity one might simply disregard this additional information.

For Poisson events, with a constant occurrence rate, one would expect half the comparisons to show reverse arrangements, and thus, $\mathrm{E}(R)=n(n-1) / 4$. The variance, in this case, is $\operatorname{var}(R)=\left(2 n^{3}+3 n^{2}-\right.$ $5 n) / 72$. It can be shown, for Poisson events with constant rate, that $R$ is approximately normal with the above mean and variance if $n \geq 10$, and tables exist for smaller $n$. Thus, for large $n$, the hypothesis of a constant rate can be rejected at the 0.05 level in favor of an increasing rate if $Z \leq-1.645$ where

$$
Z=\frac{R+0.5-\mathrm{E}(R)}{\sqrt{\operatorname{var}(R)}}
$$

The extra term 0.5 is a continuity correction. For the data of Example 3, the Mann statistic is $R=130$. Furthermore, the mean and standard deviation of $R$ are $26 \times 25 / 4=162.5$ and $\left[\left(2 \times 26^{3}+3 \times 26^{2}\right.\right.$ $5 \times 26) / 72]^{1 / 2}=22.684$. The approximate normal test is based on $Z=(130-162.5) / 22.684=-1.41$. Although this does not provide proof at the 0.05 level, it does have a p-value of 0.0793 . This is not strong evidence of a trend, but it could be considered as moderate evidence because the p-value is between 0.05 and 0.10 . It is interesting to see that even though the Laplace test did not indicate any sort of increasing trend, the Mann test detected a mild trend. The reason is that the Mann test was more influenced by the clustered values that occurred during the latter part of 1991 and continued into 1992. In other words, the last 7 event times occurred closer together than the earlier event times, resulting in a small value for $R$. For comparison, suppose only the data from the first five years of the study are used. This data set does not include the last four of the clustered data values. Excluding 1992, there were $n=22$ events with a Laplace statistic of $U=-1.20$, and a Mann statistic of $Z=0.056$, neither of which provides strong evidence of a trend. 


\section{Assumption of Independence}

The preceding subsections presented ways to investigate whether $\lambda$ is constant with respect to time, or different data sources, such as plants. Now consider the other major assumption of the basic Poisson model: that occurrences are statistically independent.

Occurrences can be dependent in many ways, and some of them can be addressed by careful thinking rather than by statistical analysis. For example, if failures of different pieces of hardware result from separate initiating causes, these occurrences can be presumed to be independent unless a common cause synchronizes the failures of the different pieces of hardware. For example, if diesel power generators share a common fuel source, then problems at the source, such as contaminated fuel, can cause all generators to fail together. If common cause failures comprise a noticeable fraction of all the failures, then the independent failures and the common cause failures should be divided into separate data sets, and the rates of failure should be estimated separately for each kind of failure.

Recall that for Poisson events with constant rate $\lambda$ the interarrival times $T_{\mathrm{i}}$ are independent and exponentially distributed with mean $1 / \lambda$. An important type of dependence would be if large interarrival times tended to occur in succession, or similarly if small ones tended to occur in succession. In other words, when interarrival times are listed in chronological order, large values tend to cluster, and similarly small values tend to cluster. For example, suppose a repair is done incorrectly several times in succession, leading to small times between failures. After the problem is diagnosed, the personnel receive training in proper repair procedures, thereafter resulting in larger times between failures. Another type of dependence would be if the values tend to alternate. That is, if small values tend to follow immediately after large values and visa versa.

A simple statistical tool for checking the assumption of independence is the Wald-Wolfowitz runs test. For example, suppose a collection of objects consists of only two types. For the sake of discussion, consider objects that have been coded as either type $A$ or type $B$. A run is an uninterrupted sequence of the same type. Consider the following sequence of ten symbols:

$A, A, B, B, B, B, A, A, A, B$

The sequence has four runs; a run of two $A$ 's, a run of four $B$ 's, a run of three $A$ 's, and finally a run of one $B$. If an $A$ makes the next symbol more likely to be another $A$, the $A$ 's tend to cluster, and similarly for the $B$ 's. In this case, the number of runs will tend to be small. On the other hand, if an $A$ makes the next symbol more likely to be a $B$, then the $A$ 's and $B$ 's tend to alternate. In this case, the number 
of runs will tend to be large. Neither clustering nor alternation of symbols would be expected if the positions of the symbols in the arrangement are chosen independently.

The Wald-Wolfowitz test is discussed by Atwood (1994) in the context of testing for randomness in a sequence of failures on demand. In that application, the data consisted of a sequence of "hits" and "misses." In the present application, the objective is to test for independence of successive interarrival times. The first step is to find the median or middle value of the interarrival times after they are ordered from smallest to largest. If there are $n$ interarrival times, then there will be a unique middle value if $n$ is odd, occurring at position $(n+1) / 2$ in the ordered array. There will be two middle values if $n$ is even, occurring at positions $n / 2$ and $(n+2) / 2$ in the ordered array. In the latter case, the median is usually defined as the average of the two middle values. In either case, there will be the same number of values greater than the median as there are less than the median. The interarrival times, considered in their original chronological order, are compared with the median, and if above the median, an $A$ is recorded, while if below, a $B$ is recorded. Any measurements that are equal to the median, as in the case of the middle observation with an odd number $n$, are omitted. The statistic of interest is the number of runs of $A$ 's and $B$ 's, denoted here by $W$. It can be shown that if $k$ is the number of $A$ 's, out of $n$ observations, then under the assumption of independence

$$
\begin{aligned}
& E(W)=\frac{2 k(n-k)}{n}+1 \\
& \operatorname{var}(W)=\frac{2 k(n-k)[2 k(n-k)-n]}{n^{2}(n-1)}
\end{aligned}
$$

and if both $k$ and $n-k$ are greater than $10, W$ is approximately normal, suggesting use of the convenient test statistic

$$
Z=\frac{W+0.5-\mathrm{E}(W)}{\sqrt{\operatorname{var}(W)}}
$$

The value 0.5 is a correction for continuity, which improves the normal approximation. For small samples, use Table $L$ of Gibbons (1976), or the exact formulas given by Bain and Engelhardt (1992).

A lower one-tailed runs test could also be used to test for a trend, because putting the $A$ 's at one end of the sequence produces fewer runs than if the $A$ 's were randomly scattered throughout the sequence. However, the Laplace and Mann tests offer more powerful tests, and therefore they would be preferred in testing for a trend. The runs test is recommended only when testing for serial dependence. 
For the data of Example 3, the interfailure times are given in Table 2.

The median is 0.14 , and the corresponding sequence of symbols is

$B, B, B, A, A, A, B, A, A, A, A, A, A, B, B, A, B, A, A, A, B, B, B, B, B, B$.

The number of runs is $W=9, k=n-k=13, \mathrm{E}(W)=14$, and $\operatorname{var}(W)=6.24$. The approximate normal test statistic is $z=-1.80$. This is not highly significant in a two-tailed test of independence. For example, a two-tailed test of size $\alpha=0.05$ would reject independence if $z<-1.96$ or $z>+1.96$. However, a two-tailed test of size $\alpha=0.10$ would reject independence if $z<-1.645$ or $z>+1.645$, indicating a moderate amount of dependence. Because $z$ is negative, the number of runs is less than would be expected if the interfailure times are independent, which indicates clustering.

Table 2. Interfailure times in chronological order of failures.

\begin{tabular}{lllllllll}
\hline 0.12 & 0.03 & 0.13 & 0.20 & 0.54 & 0.26 & 0.10 & 0.18 & 0.28 \\
0.45 & 0.15 & 0.04 & 0.34 & 0.06 & 0.11 & 0.39 & 0.11 & 0.31 \\
0.59 & 0.18 & 0.01 & 0.06 & 0.07 & 0.09 & 0.04 & 0.05 & \\
\hline
\end{tabular}




\section{MODELING VARIATION BETWEEN DATA SOURCES}

Suppose the hypothesis of a constant $\lambda$ has been rejected. What alternative model should be used? One possibility would be that $\lambda$ is a function of time. However, there are other possible choices. Advanced methods exist that can aid in the choice of an alternative model, however, this report focuses on basic methods.

Consider the case for which there are a number of distinct data sources, in no particular order, and suppose that the chi-square test has rejected the hypothesis that $\lambda$ is the same for all the data sources.

One approach is to analyze each data source separately, resulting in a separate estimate of $\lambda$ for each data source. In some cases, it might be possible to combine similar data sources, resulting in a smaller number of data sets. For example, if there is one plant that is clearly different from all the others (an outlier), it would be reasonable to treat that plant separately. Furthermore, if all the other plants are similar, their data could be pooled and treated as a single homogeneous source. After this pooling of the data, the two sources could be analyzed separately. Similar pooling procedures could be done for any number of similar data sources, and the estimates computed separately for each pooled data set.

Often, however, removal of one outlier leaves a data set that contains another outlier, and removal of that may leave a set with still another outlier. In such cases, it is simpler (i.e., it involves fewer parameters to estimate) to use the following compound model. For concreteness, the model is explained here for the example where the distinct data sets are obtained from different plants. 


\section{Gamma-Poisson Model}

The method models the variation between the plants. It is assumed that $\lambda$ varies from plant to plant, and follows a gamma $(\alpha, \beta)$ distribution. Denote by $\lambda_{\mathrm{i}}$ the value corresponding to the $i$ th plant. At this plant, conditional on the value of $\lambda_{i}$, it is assumed that events occur according to a Poisson distribution with rate $\lambda_{\mathrm{i}}$. In other words, the number of occurrences in exposure time $t_{\mathrm{i}}$ at the $i$ th plant is Poisson with mean $\lambda_{i} t_{\mathrm{i}}$. The number of occurrences $n_{\mathrm{i}}$ at a single plant, unconditional on $\lambda_{\mathrm{i}}$, has a gamma-Poisson distribution. For example, the number of failures from a single randomly selected plant would have a gamma-Poisson $(\alpha, \beta)$ distribution. This distribution, which is a form of the negative binomial distribution as discussed by Johnson, Kotz and Kemp (1992), is given by

$$
p(n)=\frac{\Gamma(\alpha+n)}{n ! \Gamma(\alpha)}(t / \beta)^{n}(1+t / \beta)^{-(\alpha+n)} \quad n=0,1,2, \ldots
$$

Atwood (1984) discusses the method of maximum likelihood estimation for this model, and points out that the resulting maximum likelihood estimates (MLEs) are not given by explicit formulas. Note that Atwood uses the reciprocal $1 / \beta$ rather than $\beta$, and also recommends a different parameterization which, in the present notation, is $\alpha$ and $\mu=\alpha / \beta$. A numerical iteration method for computing the MLEs, written in the SAS language, is given in Appendix A as the program gpmle.

Let us apply this to the data used in Figure 4.

If the data from all the plants were pooled, and the rate assumed the same for all plants, the MLE of a common rate would be $\hat{\lambda}=92 / 98.02=0.94$. For the combined count from all plants, the Bayes posterior distribution for $\lambda$ based on the prior density, which is proportional to $\lambda^{-1 / 2}$, would be gamma $(92.5,98.02)$. The mean of the Bayes posterior distribution is $92.5 / 98.02=0.944$, and a Bayes $90 \%$ interval based on this distribution is $(0.79,1.11)$. The calculations were performed by the SAS macro pbayes in Appendix A. However, the chi-square statistic for testing the equality of the $\lambda_{i}$ 's for the 23 plants strongly supports the claim that the $\lambda_{i}$ 's are not all equal. We find that $X^{2}=43.83$ with $\nu=22$ degrees of freedom, which has a corresponding p-value of 0.004 .

Thus, we consider the gamma-Poisson model, which allows the value of $\lambda$ to vary from plant to plant. In this framework, and using the data from Figure 4, the MLE's based on the gamma-Poisson distribution are $\hat{\alpha}=4.67$ and $\hat{\beta}=4.78$. This was found using the SAS macro gpmle, in Appendix A. If we use these estimates in place of $\alpha$ and $\beta$ in the gamma distribution, the estimated mean of the gamma $(\alpha, \beta)$ distribution is $\hat{\mu}=\hat{\alpha} / \hat{\beta}=0.98$, and estimates of the 0.05 th and 0.95 th quantiles of the 
$\operatorname{gamma}(\alpha, \beta)$ distribution are $\chi_{0.05}^{2}(2 \times 4.67) /(2 \times 4.78)=0.37$ per year and $\chi_{0.95}^{2}(2 \times 4.67) /(2 \times 4.78)=1.82$ per year. Thus, a $90 \%$ interval for $\lambda$ is $(0.37,1.82)$.

\section{Empirical Bayes Estimates}

Having estimated the overall distribution of $\lambda$, we now inquire about the rate $\lambda_{i}$ at the $i$ th plant. The model regards the value $\lambda_{\mathrm{i}}$ as randomly selected from a gamma $(\alpha, \beta)$ distribution, and the $n_{\mathrm{i}}$ events counted in exposure time $t_{\mathrm{i}}$ were generated from a Poisson distribution with mean $\lambda_{\mathrm{i}} t_{\mathrm{i}}$. Of course, $\alpha$ and $\beta$ are unknown, but we have calculated MLE's $\hat{\alpha}$ and $\hat{\beta}$ using data from all of the plants. The simplest approach is to act as if the estimates were the true values. In other words, let the prior distribution of $\lambda_{i}$ be gamma $(\hat{\alpha}, \hat{\beta})$, and find the posterior distribution of $\lambda_{i}$ by updating the prior with the data from the $i$ th plant. This is the usual Bayes method for estimating $\lambda_{i}$, except that the prior parameters are estimated using data from all plants. This leads to the name "empirical Bayes estimation."

More sophisticated methods exist which account for the variability in the estimators $\hat{\alpha}$ and $\hat{\beta}$. In particular, Kass and Steffey (1989) give a simple first-order correction. This method is also discussed by Martz (1994). This approach, which leaves the plant-specific mean unchanged, adjusts the posterior variance of $\lambda_{i}$, thus accounting for the uncertainty due to having estimated $\alpha$ and $\beta$ from data. A SAS program to implement the method in given is Appendix A as gpempb, and the technical details of the method are given in Appendix B. The effect on the mean of the empirical Bayes distribution is small, and if the number of data sources is large, the effect of the adjustment on the variance is also small.

Before showing a graph of empirical Bayes results, we first give a graph of ordinary (non-empirical) Bayes results. Figure 7 shows the Bayes mean and $90 \%$ interval for each plant, based on the noninformative prior distribution and the plant-specific data.

This figure is analogous to Figure 5, with Bayesian estimates and intervals replacing the corresponding MLEs and confidence intervals. Figures 5 and 7 appear similar, although the Bayes point estimates are pulled from the MLEs toward the prior mean, which is $+\infty$ for the improper gamma prior with $\alpha=$ 0.5 and limiting value $\beta=0$. Also, the Bayes intervals are slightly shorter than the corresponding confidence intervals.

Figure 7 is given for the purpose of comparison with the empirical Bayes method, and is not intended as a competing method. Only if the data sources are believed to have no relation to each other 


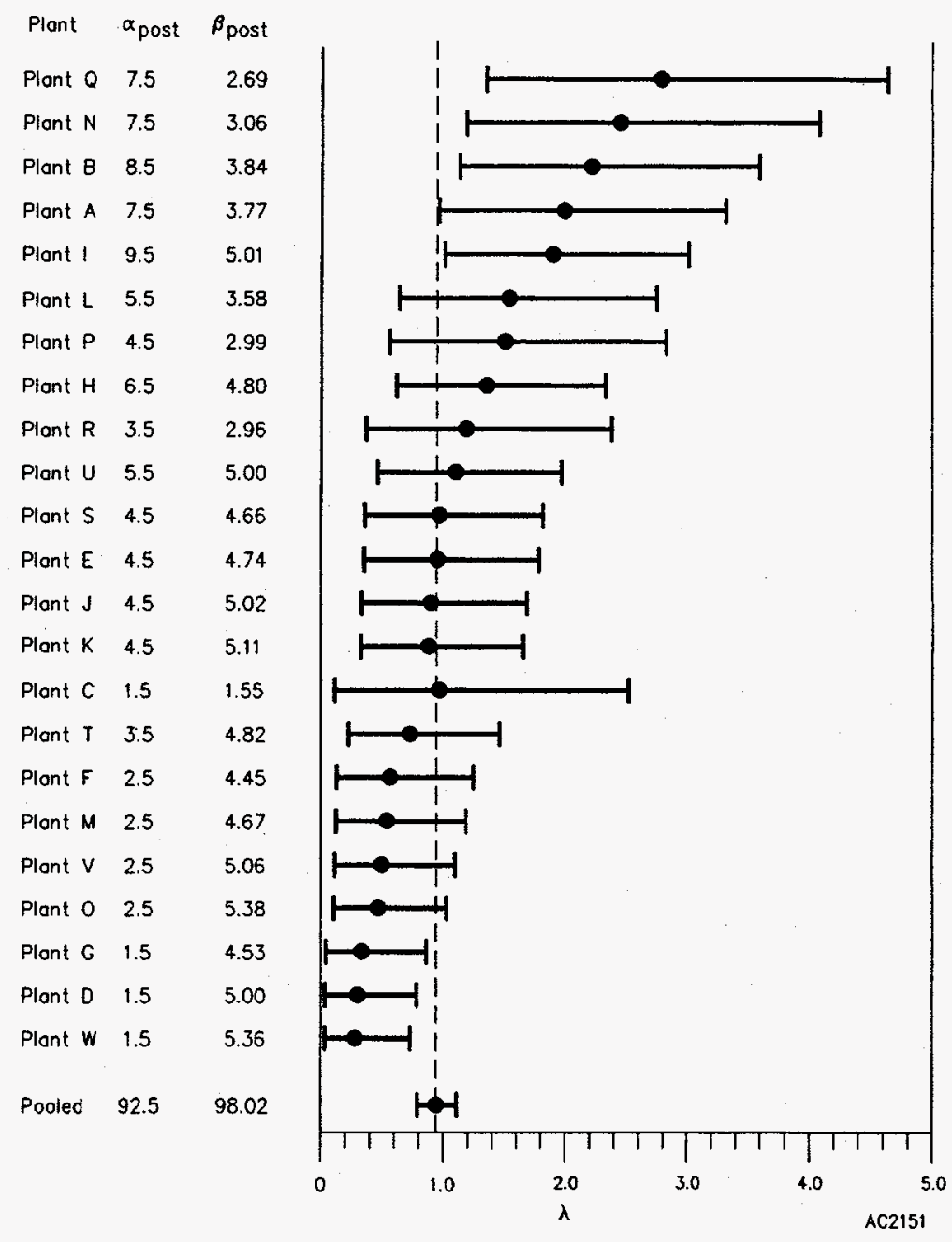

Figure 7. Bayes means and $90 \%$ intervals for $\lambda_{\mathrm{i}}$, with each plant estimate based on a noninformative prior distribution.

(such as data from different kinds of components, for example), would separate analyses like those of Figure 7 be used. If the data sources (plants, in this example) are all believed to behave as if their $\lambda$ 's were drawn randomly from some population, and if no prior reason exists to assign any particular $\lambda$ to any particular data source, then the empirical Bayes method is appropriate.

For example, Plant A had 7 failures in exposure time 3.77 years, and consequently its empirical Bayes distribution is gamma with estimated parameters $\hat{\alpha}+7=11.67$ and $\hat{\beta}+3.77=8.55$. The point estimate of $\lambda_{A}$ is $11.67 / 8.55=1.36$ events per year. The parameter values for the empirical Bayes posterior distribution, after the variance adjustment described in Appendix $\mathrm{B}$, are $\alpha_{\mathrm{adj}}=10.06$ and $\beta_{\mathrm{adj}}$ $=7.38$. The corresponding $90 \%$ empirical Bayes confidence interval for Plant $\mathrm{A}$ is $(0.74,2.14)$. For 
comparison, the Bayes point estimate and $90 \%$ interval for Plant A based on the noninformative prior are, respectively, $7.5 / 3.77=1.99$ and $(0.96,3.32)$.

Figure 8 shows the empirical Bayes point estimates and $90 \%$ intervals for all plants in the study. In general, the intervals do not vary as much from each other as in Figure 7. The empirical Bayes mean for each plant is pulled toward the prior mean of 0.98 , and the intervals are somewhat shorter, resulting from the use of an estimated prior rather than a noninformative prior.

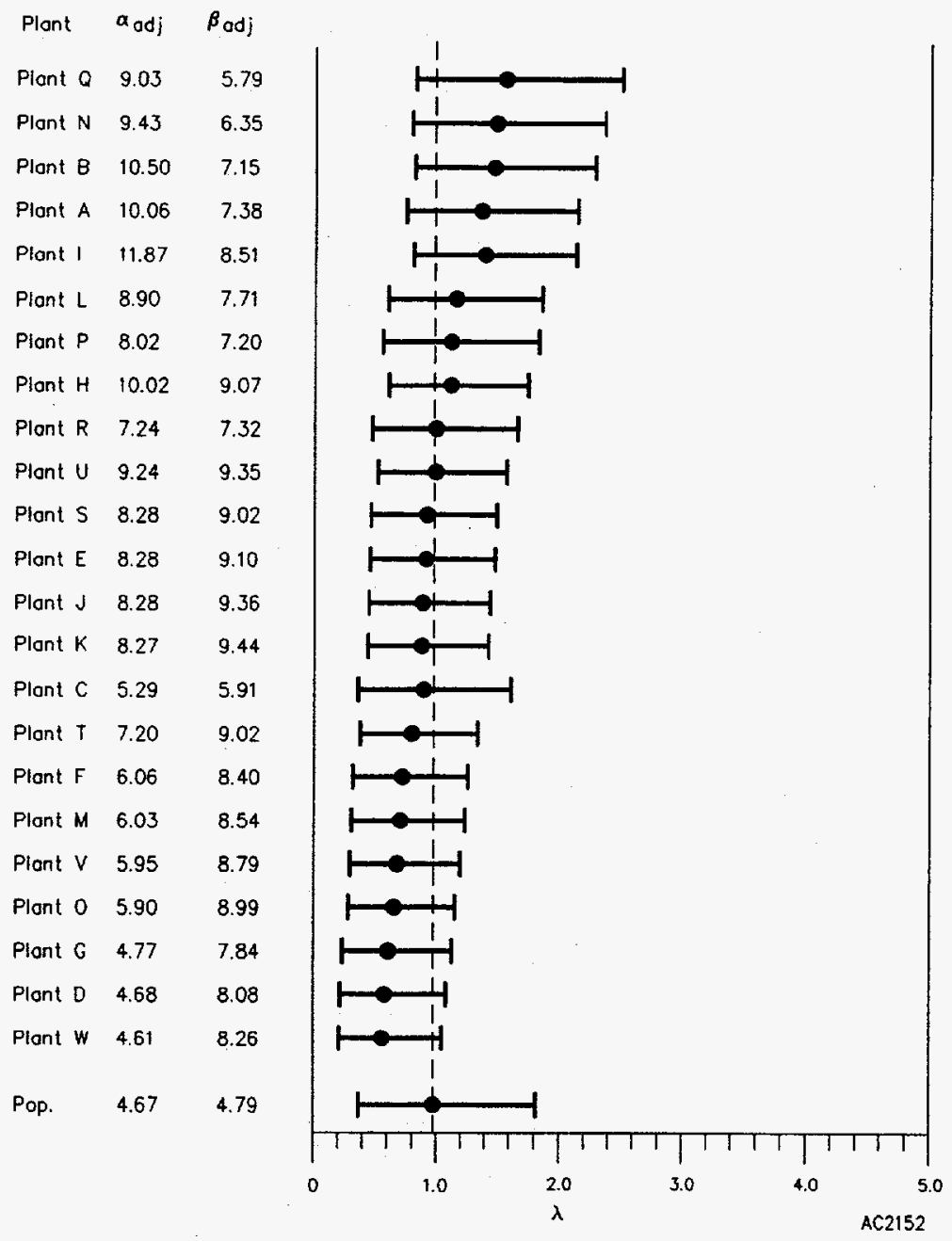

Figure 8. Empirical Bayes means adn $90 \%$ intervals for $\lambda_{i}$, with variance adjustment.

Notice that the Bayes estimate of $\lambda$ for Plant $\mathrm{C}$ does not fit the pattern of monotonicity which was imposed on the estimates in Figure 5. Generally, Bayes estimates based on smaller amounts of data will be influenced more strongly by the prior distribution than those involving larger amounts of data. In the case of Plant $\mathbf{C}$, there was only one observed failure and the exposure time was only 1.55 years. The high degree of uncertainty for this data set is reflected in Figure 5 in the form of a relatively lengthy 
confidence interval for $\lambda$. Thus, the Bayes point estimates of $\lambda$ for Plant $C$ in Figures 7 and 8 are pulled more strongly toward the respective prior means than estimates for the adjacent plants (Plants $\mathrm{K}$ and $\mathrm{T}$ ), which involved higher counts and longer exposure times.

With either method, the degrees of freedom are non-integer valued. Some books require that the degrees of freedom be integers. However, this is unnecessarily restrictive because the shape parameter of a gamma distribution can be any positive real value. The quantiles can be obtained by interpolation from ordinary $\chi^{2}$ tables. Also, some of the commonly used software such as SAS will compute $\chi^{2}$ quantiles for non-integer degrees of freedom.

Two points should be mentioned. First, the empirical Bayes method only models, or quantifies, the variation. It does not explain the causes. The poor showing of Plant $\mathrm{Q}$ may be the result of worse procedures, worse equipment, or some other cause. An investigation into such causes goes beyond the scope of the empirical Bayes data analysis.

Second, we should ask whether the variation was modeled correctly. The method assumed a gamma prior distribution and then mechanically found plant-specific posterior means and intervals. This is appropriate only if the assumed model fit the data adequately.

One way to address this question is to ask whether the data counts are consistent with the gammaPoisson model. Consider Plant $Q$ as an example. The gamma-Poisson probability of 7 failures in exposure time 2.69 is given as $p(7)$ by Equation (B-3) of Appendix B. The probability of 7 or more failures is $\Sigma_{i} p(i)$ where $i$ runs from 7 to $\infty$. Of course, a better way to evaluate this is to compute 1 $\Sigma_{p} p(i)$ where $i$ runs from 0 to 6 . The SAS macro gpempb, used the estimates $\hat{\alpha}$ and $\hat{\beta}$ for the unknown $\alpha$ and $\beta$, and evaluated the probability of 7 or more failures as 0.048 . This is a $p$-value, the probability that the data would appear as extreme as observed if the model assumptions are correct. From 20 plants, we would expect about one to have a p-value as small as $1 / 20=0.05$. Similarly, for 23 plant, we would expect about $1 / 23=0.043$. In fact, out of 23 plants, the p-value of 0.048 , as noted above for Plant $Q$, is the smallest. The technical details are more complex than indicated here, but it appears that there is no strong evidence against the assumption of a gamma prior. 


\section{REVIEW AND GUIDELINES}

This report consists primarily of a collection of tools that can be applied as needed, not a set of procedures that tell the reader what to do. Now that the tools have been presented, however, it is appropriate to review them and to give some general guidelines on when to use them. This also helps to show how they relate to each other.

\section{The Basic Model and Its Data Analysis}

The Poisson model makes some basic assumptions: the probability that an event occurs in a specified short interval of time is approximately proportional to the length of the interval, with the same rate $\lambda$ regardless of the location of the interval; the probability that more than one event occurs in a specified short interval is negligible relative to the length of the interval; occurrences of events are statistically independent in disjoint intervals of time. The analyst must think about the meaning of these assumptions for the particular data set under consideration. Much of the effort in data analysis involves checking the assumptions and adapting the methods to match the assumptions that seem to be true.

If the assumptions of the Poisson model are believed to be true, the main issue is to estimate $\lambda$. Formulas have been given for the point estimate (the maximum likelihood estimate) and confidence interval. A SAS macro pconfint has also been given to calculate these. If preferred, a Bayesian distribution may be found, yielding a point estimate (the mean of the distribution) and a Bayes interval. The SAS macro pbayes calculates these. As long as the Bayesian analysis uses the noninformative prior, the confidence interval and Bayes interval will be numerically similar, though not identical.

Which analysis to use is partly a matter of the analyst's and customer's philosophy and taste. When $n$ is not very large, the Bayes estimate and limits for a Bayes interval will be visibly pulled toward the mean of the prior density, which is infinite for a noninformative gamma prior. Thus, the limits tend to be high with sparse data sets. Also, some analysts prefer not to use Bayes intervals because they lack the usual relative frequency interpretation of confidence intervals.

The choice of Bayesian or non-Bayesian analysis is also partly a matter of simplicity. Confidence intervals are related naturally to hypothesis tests, and are therefore natural to use when checking the model assumptions. Most PRA work requires a Bayesian approach, and therefore the final product of a PRA-oriented analysis may be a Bayesian distribution. To propagate the uncertainty in $\lambda$ through a complicated system, PRA computer codes such as IRRAS (Russell et al. 1992, 1993) use Monte Carlo 
simulation, sampling $\lambda$ from its Bayes distribution. The required inputs for IRRAS, based on the posterior distribution have been discussed in the report.

\section{Checking the Assumptions of the Poisson Model}

To check the assumptions of the simple model, graphical methods and statistical tests have been presented. Three questions were addressed:

- Is $\lambda$ the same for a number of distinct data sources?

- Is $\lambda$ constant over time for the same data source?

- Are occurrences over time independent?

For the first question, the methods given were a plot of side-by-side confidence intervals, and chisquare tests with $1 \times c$ contingency tables. The SAS macro pconfint performs all the necessary calculations. For the second question, the methods given were a cumulative plot of event occurrences versus time, and two statistical tests. The SAS program Mann is provided in Appendix A. The question of independence may only require careful thinking, not statistical analysis. However, the runs test applied to interarrival times was presented as a test for serial dependence.

\section{An Extension of the Poisson Model}

Suppose there are distinct, but inherently similar, data sources such as systems of similar design at different facilities. If a chi-square test shows that the data sources have different values of $\lambda$, this variation can often be modeled by assuming that $\lambda$ has a gamma distribution. The parameters of this distribution can be estimated by maximum likelihood; the SAS macro gpmle can be used for this. Once the gamma distribution on $\lambda$ has been estimated, it can be used as a prior distribution for each of the different data sources.

This is a simple implementation of the empirical Bayes method. A more refined implementation, carried out by means of the SAS macro gpempb, accounts for the uncertainty in the parameter estimates, thus yielding slightly longer intervals. This macro also checks whether the data sources are adequately modeled by the gamma-Poisson model. 


\section{REFERENCES}

Ascher, Harold. and Feingold, Harry, 1984, Repairable Systems Reliability, New York: Marcel Dekker.

Atwood, Corwin L., 1981, "Tests of a Simple Multinomial Hypothesis when the Sample is Not Large," ASA Proceedings of the Stat. Computing Sec., pp. 130-134.

Atwood, Corwin L., 1982, "User's Guide to HOMOG: A Computer Program for Investigating Homogeneity of Poisson Data Sources," EGG-EA-5726.

Atwood, Corwin L., 1993, Hits per Trial: Basic Analysis of Binomial Data, (Draft) EGG-RAAM-11041.

Atwood, Corwin L., 1984, "Approximate Tolerance Intervals, Based on Maximum Likelihood Estimation," Journal of the American Statistical Association, 79, 386, pp. 459-465.

Bain, Lee J. and Engelhardt, Max, 1992, Introduction to Probability and Mathematical Statistics, 2nd ed., Boston: PWS-Kent.

Box, George E. P., and George C. Tiao, 1973, Bayesian Inference in Statistical Analysis, Reading, MA: Addison-Wesley.

Cleveland, William S., 1985, The Elements of Graphing Data, Monterey, CA: Wadsworth Advanced Books and Software.

Cox, D. R. and P. A. W. Lewis, The Statistical Analysis of Series of Events, 1978, London: Chapman and Hall.

D'Agostino, R. B., and M. A. Stephens, 1986, Goodness-of-Fit Techniques, New York: Marcel Dekker.

Gibbons, Jean D., 1976, Nonparametric Methods for Quantitative Analysis, New York: Holt, Rhinehart, and Winston.

Hollander, Myles, and Douglas A. Wolfe, 1973, Nonparametric Statistical Methods, New York: John Wiley \& Sons. 
Johnson, Norman L., Samuel Kotz, and Adrienne Kemp; 1992, Univariate Discrete Distributions, New York: John Wiley \& Sons.

Kass, Robert E. and Duane Steffey, 1989, “Approximate Bayesian Inference in Conditionally Independent Hierarchical Models (Parametric Empirical Bayes Models)," Journal of the American Statistical Association, 84, pp. 717-726.

Koehler, K. J., and K. Larntz, 1980, “An Empirical Investigation of Goodness-of-Fit Statistics for Sparce Multinomials," Journal of the American Statistical Association, 75, pp. 336-344.

Martz, H. F., 1994, "Modern Empirical Bayes Failure Rate Estimation in the Poisson Distribution with NRC Applications," Los Alamos National Laboratory Report LA-UR-94-661, Los Alamos, New Mexico.

Martz, Harry F., and Ray A. Waller, Bayesian Reliability Analysis, Malabar, FL: Krieger, 1991, Section 7.6.

Meyer, Paul L., 1970, Introductory Probability and Statistical Applications, Reading, Massachusetts: Addison-Wesley Publishing Co.

Roscoe, J. T., and J. A. Byars, 1971, "An Investigation of the Restraints with Respect to Sample Size Commonly Imposed on the Use of the Chi-square Statistic," Journal of the American Statistical Association, 66, pp. 755-759.

Russell, K. D. et al., 1992, Integrated Reliability and Risk Analysis System (IRRAS) Version 4.0: Reference Manual, NUREG/CR-5813, EGG-2664, Vol. 1.

Russell, K. D., C. L. Atwood, M. B. Sattison, and D. M. Rasmuson, 1993, SAPHIRE Technical Reference Manual: IRRAS/SARA Version 4.0, NUREG/CR-5964, EGG-2692.

SAS Institute Inc., 1988, SAS/STAT User's Guide, Release 6.03 Edition, Cary, NC. 
Appendix A

\section{SAS Programs}


A-2 


\section{Appendix A}

\section{SAS Programs}

This section contains SAS programs to perform analyses discussed earlier in this document.

\section{MACRO PCONFINT}

$\%$ macro pconfint (datain, id, events, expos, dotest);

* datain is name of data set

* id is name of record identifier in the data set

* events is name of variable containing event counts, in the data set

* expos is name of variable containing exposure time.

* dotest is $T$ if chi-squared test of homogeneity is to be performed

* $\quad F$ otherwise. Do not enclose this letter in quotation marks.

$\star$

* WARNING: This macro creates temporary data sets whose names begin with $x x x$.

* The user should avoid such names, or accept that the macro may change them.

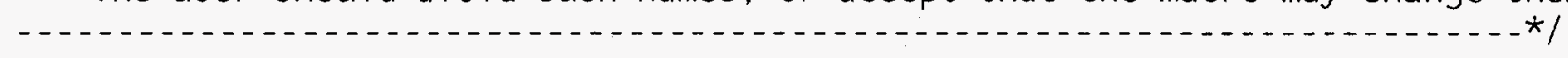

DATA xxxpc1; set \&datain;

* Here I sum two columns:

proc summary:

var \&events \&expos:

output out $=x \times x p c 2 \quad$ SuM=evsum exsum; run;

* Here I get and use the sums:

data; set $\times x \times p c 1$ end=endindiv;

if $N=1$ then set $x \times x p c 2$;

format lamlo lamhat lamup el0. :

if (\&expos > 0) then do;

eev $=$ evsum*\&expos/exsum;

chisq $=($ (\&events-eev $) \star \star 2) /$ eev :

numer $10=0$ :

if \&events $>0$ then numer $10=\operatorname{cinv}(.05,2 *$ \&events $)$

numerup $=\operatorname{cinv}(.95,2 *($ \&events +1$))$;

lamhat $=$ \&events $/$ \&expos:

1amlo $=$ numer $10 /(2 * \& \operatorname{expos})$;

lamup $=$ numerup $/(2 * \& \operatorname{expos})$ :

output:

end:

else do:

lamhat $=$. : 
lam1o =.
lamup =.
chisq = $;$
output

end;

if endindiv then do;

\&id $=$ 'Tota $]^{\prime}$

\&events = evsum

\&expos $=$ exsum

chisq $=$

numer $10=0$

if \&events $>0$ then numerlo $=\operatorname{cinv}(.05,2 *$ \&events $)$;

numerup $=\operatorname{cinv}\left(.95,2^{*}(\right.$ \&events +1$\left.)\right)$;

lamhat $=$ \&events $/$ \&expos;

$1 \mathrm{am} 70=$ numer $10 /(2 *$ \&expos $)$;

1amup $=$ numerup $/(2 * \& \operatorname{expos})$ :

output:

end:

run;

proc print:

var \&id \&events \&expos 1amlo lamhat lamup chisq;

run:

\% if "\&dotest"="T" \%then \%do;

title ' ':

proc summary :

var chisq:

output out=temp sum $=\mathrm{N}=$ numobs :

run:

data; set:

drop type freq_:

pvalue $=1^{-}$- $\operatorname{probchi}$ (chisq, numobs-1);

run:

proc print; run;

\%end;

\%mend pconfint :

\section{MACRO PBAYES}

\%macro pbayes(datain, id, aprior, bprior, events, time):

* Finds Bayes 90\% interval for Poisson parameter, with time-censored data

* First argument = name of data set containing count data

* Second argument $=$ name of variable with record identifier in input data set 
* Third argument $=$ shape parameter of prior distr. . a number or variable name

* Fourth argument $=1 /$ (scale parameter) for prior distr. , a number or var. name

* Fifth argument $=$ name of variable with count of events, in input data set

* Sixth argument = name of variable with exposure time, in input data set

*

* To enter noninformative parameter, let aprior $=5$, bprior $=0$ :

* To change content of interval, change arguments to gaminv

$\star$

DATA; set \&datain;

apost $=$ \&aprior + \&events

bpost $=$ \&bprior + \&time

1am1o $=$ gaminv $(.05$, apost $) /$ bpost

lamean $=$ apost $/$ bpost

Tamup $=$ gaminv $(.95$, apost $) /$ bpost

run:

proc print;

var \&id apost bpost lamlo lamean lamup;

run;

\%mend pbayes:

\section{PROGRAM MANN}

data def.renew; infile 'gnplot.dat';

input time:

rentime=time-lag(time); *renewal time;

if $n_{-}=1$ then rentime=time- 0 ;

run:

proc print; run;

\%macro mann(renewvar):

/*

This program performs the Mann test for trend to see if the number of events counted in time $t$ is a renewal process, with the alternative that the times between occurrences tend to become longer as $t$ increases. *1

* reset dimension if more than 100 events:

data zzztemp: set nobs=nobs end=eof:

keep trials mann e_mann v_mann z_mann p_value:

array rtime[100]:

retain rtime:

rtime $\left[n_{-}\right]=\&$ renewvar:

if eof then do; 
mann=0 ;

do marker=1 to (nobs-1);

do times $=($ marker+1) to nobs:

if rtime[marker] < rtime[times] then mann=mann+1; end;

end;

trials=nobs:

e mann=trials*(trials -1$) / 4$;

$\checkmark$ mann $=(2 * \operatorname{trials} * * 3+3 * \operatorname{trials} * * 2-5 * \operatorname{trials}) / 72$;

$z-\operatorname{mann}=(\operatorname{mann}+.5-e$ mann $) / \operatorname{sqrt}(v$ mann $)$;

p_value =probnorm ( $\bar{z}$ mann $)$;

$i \bar{f} p_{-} v a l u e>.5$ then $p_{-}$value $=1-p_{-}$value:

$$
\text { outpūt: }
$$

end;

run:

proc print; var mann e_mann p_value trials v_mann z_mann; run:

\%mend mann;

i* Next program uses event times to calculate between-event times, then calls above macro.*/

\%mann(rentime)

\section{MACRO GPMLE}

\%macro gpmle(datain, dataout, evnt, time);

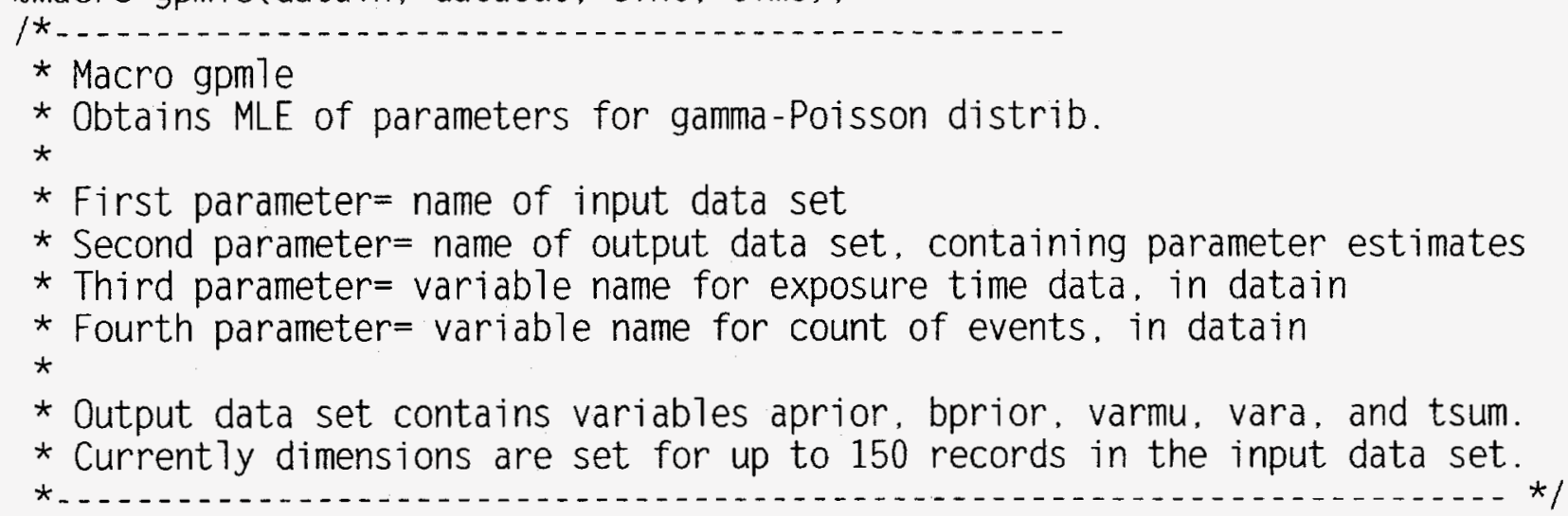

data \&dataout; set \&datain end=eof nobs=nobs:

keep aprior bprior varmu vara tsum;

array $t[150]$;

array e[150]:

retain $t e$ :

$t[n]=\&$ time:

$e\left[n_{-}\right]=\& e v n t$; 
* Sum events and times:

esum + \&evnt;

tsum + \&time:

if eof then do;

*Bound ML Estimates:

beta $70=0$;

betahi=tsum;

beta = betahi ;

link derivsub: *calc value of deriv of LogLik function;

if (deriv $L L>0)$ then do;

file print notitle:

put '..............

put ' --Reverting to non-informative prioor-.-';

file log:

aprior $=$ esum +0.5 ;

bprior = tsum :

mu = aprior/bprior:

varmu $=0$;

vara $=0$;

end;

else do;

* Bisection search for MLEs:

do until (betahi-betalo<.00001*beta10):

beta $=($ betalo+betahi $) / 2$;

link derivsub:

if deriv $L L>0$ then betalo=beta:

else betahi=beta:

put betalo $=$ betahi $=\operatorname{derivLL}=$;

end:

* MLEs found;

* Now save MLEs of empirical Bayes prior

* and calculate values for Kass-Steffey adjustment.

* Do this with parameterization in terms of alpha and mu;

aprior = alphahat;

bprior = beta;

mu $=$ aprior/bprior:

* Find information matrix, in terms of aprior and mu:

sum $1=0 ; \operatorname{sum} 2=0 ; \operatorname{sum} 3=0$;

do $i=1$ to nobs:

if $e[i]>0$ then do $j=0$ to $e[i]-1$;

sum1 $=$ sum $1+1 /($ aprior $+j) \star \star 2 ;$

end;

sum2 $=$ sum2 + mut $^{\star} t[i] /\left(\right.$ aprior* $\left(\right.$ aprior $\left.\left.+m u^{\star} t[i]\right)\right)$;

sum3 $=$ sum3 + aprior*t $(i) /\left(m u^{*}\left(\right.\right.$ aprior $\left.\left.+m u^{*} t[i]\right)\right)$;

end;

info11 = sum1 - sum2:

info22 = sum3; 
if (infoll $<=0$ ) then do;

file print notitle;

put ' $\star \star \star \star \star E R R O R ! ! '$ sum $1=$ sum $2=$ sum $3=$ infol1 $=$ infor2 $=$;

file log:

info11 = 0 ;

end;

vara $=1 /$ infoll:

varmu $=1 /$ info 22 ;

sdva $=\operatorname{sqrt}($ vara $)$;

sdvmu=sqrt (varmu);

1 am7o $=\operatorname{gaminv}(0.05$, aprior $) /$ bprior:

1amup $=\operatorname{gaminv}(0.95$, aprior $) /$ bprior:

lamean = mu;

file print notitle;

put;

put

-Fitted Prior-............

putexpr="MLE Prior from "|| left(esum) ||" events in "||

left(tsum) ||" exposure time units:":

put putexpr:

put aprior $=$ bprior $=1 \mathrm{amlo}=1 \mathrm{amean}=1 \mathrm{amup}=$ :

put $s d v a=$ vara $=s d v m u=$ varmu $=$;

put

file $10 \mathrm{~g}$;

end:

output;

end:

return:

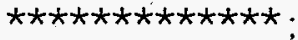

derivsub:

${ }^{*}$ calc derivative wrt beta of loglike sum and put value in variable derivLL;

if $\operatorname{bet} a=0$ then derivLL=0:

else do:

${ }^{*}$ calc alphahat:

link ahatsub;

derivLL=0;

do $i=1$ to nobs:

derivLL=derivLL+digamma (a]phahat+e[i])-digamma (alphahat)

end: $-\log (1+\mathrm{t}[\mathrm{i}] /$ beta $)$;

end:

return:

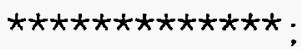

ahatsub:

${ }^{*}$ calc alpha hat:

sum $1=0$; sum $2=0 ;$ sum $3=0$;

do $i=1$ to nobs;

suml $1=$ suml $1+e[i]$; 
sum2 $=\operatorname{sum} 2+e[i] * t[i] /($ beta $+t[i])$;

sum3 $=$ sum3 $+\mathrm{t}[i] /($ beta $+\mathrm{t}[\mathrm{i}])$;

end:

a)phahat=( sum1 - sum2) $/$ sum3:

return;

run;

\%mend:

\section{MACRO GPEMPB}

\%macro gpempb(datain, dataemb, id, evar, tvar);

$1 *$

Finds Bayes posterior mean and $90 \%$ interval for binomial parameter.

for each record.

Also finds whether any record is in tail of the prior distr.

datain = input data set with event data. Typically, this is the same data set that was used as an input to gpmle.

dataemb = input data set containing empirical Bayes estimates. Typicaliy, this

is dataout from gpmle. The variable names must be aprior, bprior, varmu,

vara, and tsum.

id = name of variable containing record identifier in datain

evar = name of variable with event counts

tvar = name of variable with exposure times

For example, if 'mydata' has variables 'plant', 'events', and 'times'.

an empirical Bayes analysis by plant could be performed by the two lines:

\%bbinmle (mydata, datatmp, events, times):

\%bbempb (mydata, datatmp, plant, events, times);

data; set \&datain NOBS=nobs end=last:

keep \&id apost bpost 1amlo mupost 1amup adjapost adjbpost adjlamio adjlamup leftprob rghtprob :

retain muprior:

if $N=1$ then do:

$\overline{s e} \bar{t}$ \&dataemb;

muprior=aprior/bprior:

end:

apost $=$ aprior + \&evar:

bpost $=$ bprior + \&tvar:

mupost $=$ apost $/$ bpost;

$1 \mathrm{am} 10=\operatorname{gaminv}(.05$, apost $) /$ bpost ;

lamup = gaminv $(.95$, apost $) /$ bpost :

* Next three lines give (d mupost)/(d aprior) and (d mupost)/(d muprior) ;

* Note, derivs are parameterized in terms of alpha and mu, not alpha and beta, for greater stability in estimates; 


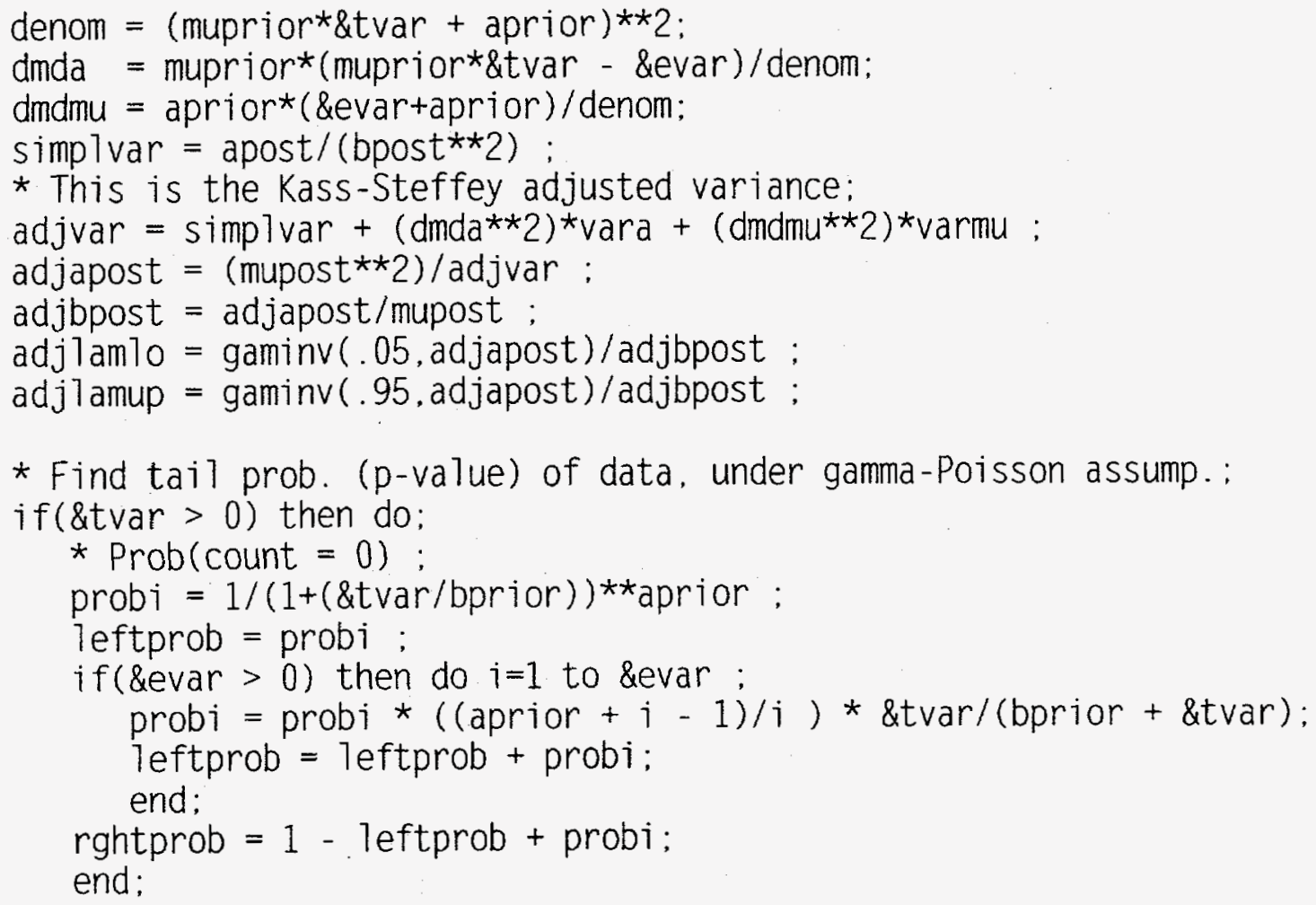


Appendix B

\section{Technical Details}

B-1 
B-2 


\section{Appendix B}

\section{Technical Details}

\section{CONFIDENCE INTERVALS AND BAYES INTERVALS}

In this section, confidence intervals and Bayes intervals for a rate parameter $\lambda$, based on having observed $n$ events, are derived. The frequentist approach, leading to confidence intervals, treats $\lambda$ as fixed and the data value $n$ as random, while the Bayes approach treats $\lambda$ as random and the data value $n$ (after it has been observed) as fixed. Nevertheless, reasonable people have adopted both approaches and obtained credible answers. Therefore, it is not too surprising that there is a numerical relation between confidence intervals and Bayes intervals.

A more detailed discussion, comparing the general philosophies of frequentist and Bayesian approaches is given by Atwood (1994), in the context of the binomial distribution. For the remainder of this appendix only the derivation of the intervals will be considered.

For simplicity of presentation, the desired confidence level is always assumed to be $90 \%$, corresponding to tail probabilities of 0.05 . The subscripts 'conf' and 'Bayes' will be used to distinguish between confidence limits and Bayes limits.

\section{Confidence Intervals}

First, we note that exact $90 \%$ confidence cannot be achieved, because the Poisson random variable is discrete. The desired statement at the lower end is that

$$
P\left(\lambda<\gamma_{\text {conf }, L}\right)=0.05
$$

for all $\lambda$. Here $\lambda_{\text {conf,L }}$ is the lower end of the confidence interval, calculated from the random data. The number of events observed in a fixed time interval of length $t$ can only take nonnegative integer values. Therefore, no matter how cleverly we adjust the formula for the lower limit $\lambda_{\text {conf }, L}$, it can only take a countable number of values, and it is therefore not possible to make Equation (B-1) exactly true for all $\lambda$. Instead, it is customary to adopt a conservative definition, and require instead that 


$$
P\left(\lambda \leq \lambda_{\text {conf }, L}\right) \leq 0.05
$$

and similarly for the upper point we require that

$$
P\left(\lambda \leq \lambda_{\text {conf }, U}\right) \leq 0.05
$$

Because $P\left(\lambda_{\text {conf }, \mathrm{L}} \leq \lambda \leq \lambda_{\text {conf }, \mathrm{U}}\right)=1-\left[P\left(\lambda<\lambda_{\text {conf }, \mathrm{L}}\right)+P\left(\lambda_{\text {conf }, \mathrm{U}}>\lambda\right)\right] \geq 0.90$, this leads to an interval with at least $90 \%$ confidence of covering the true value of $\lambda$.

Conservative confidence limits can be constructed for a discrete statistic from cumulative probabilities (Bain and Engelhardt, 1992, p. 373). In general, suppose $S$ is a statistic whose distribution depends on a parameter $\theta$. If the cumulative distribution function $\mathrm{G}(s ; \theta)=\mathrm{P}[S \leq s ; \theta]$ is decreasing in $\theta$ for each fixed $s$, then a conservative lower $95 \%$ confidence limit for $\theta$ is a solution to the equation $\mathrm{P}[S$ $<s ; \theta]=0.95$, and a conservative upper $95 \%$ confidence limit for $\theta$ is a solution to the equation $\mathrm{P}[S$ $\leq s ; \theta]=0.05$. Note that if $S$ is integer-valued, then $\mathrm{P}[S<s]=\mathrm{G}(s-1 ; \theta)$, so that a lower $95 \%$ confidence limit is defined by $0.95=\mathrm{G}\left(s-1 ; \theta_{\mathrm{L}}\right)$, and an upper $95 \%$ confidence limit for $\theta$ is defined by $0.05=\mathrm{G}\left(s ; \theta_{\mathrm{U}}\right)$. These results will be used in the following discussion to derive conservative upper and lower confidence limits for the rate parameter $\lambda$. This will also involve $\chi^{2}$ quantiles, and in the notation used below, $\chi_{\mathrm{p}}^{2}(\nu)$ is the $p$ th quantile of the $\chi^{2}$ distribution with $\nu$ degrees of freedom.

As mentioned in the body of this report, confidence limits for $\lambda$ can be expressed in terms of $\chi^{2}$ quantiles. This results from a relationship between the Poisson and $\chi^{2}$ distributions. Specifically, if $N$ is Poisson with mean $\mu$ and $X$ is $\chi^{2}$ with $2 n$ degrees of freedom, then $\mathrm{P}[N<n ; \mu]=\mathrm{P}[X>2 \mu ; 2 n]$ (Bain and Engelhardt, 1992, p. 286). Thus, $0.95=\mathrm{P}\left[N<n ; \mu_{\mathrm{L}}\right]$ and $0.05=\mathrm{P}\left[N \leq n ; \mu_{\mathrm{U}}\right]=\mathrm{P}[N$ $<n+1 ; \mu_{U}$ l if and only if $\mu_{\mathrm{L}}=\chi_{0.05}^{2}(2 n) / 2$ and $\mu_{U}=\chi_{0.95}^{2}(2 n+2) / 2$. Because $\mu=\lambda t$, the limits for a conservative $90 \%$ confidence interval for $\lambda$ are

$$
\begin{aligned}
\lambda_{\text {conf }, L} & =\chi_{(0.05)}^{2}(2 n) /(2 t) \\
\lambda_{\text {conf }, U} & =\chi_{0.95}^{2}(2 n+2) /(2 t)
\end{aligned}
$$

\section{Bayes Intervals}

Now consider the Bayesian interval, with $\lambda$ treated as a random variable. We will derive an equation that is very similar to Equation (B-2), only the degrees of freedom will be different; this will allow a direct comparison of the Bayes interval and the confidence interval. 
As mentioned previously, the rate parameter $\lambda$ is assumed to have a prior distribution, which is $\operatorname{gamma}(\alpha, \beta)$,

$$
f(\lambda)=\frac{\beta^{\alpha}}{\Gamma(\alpha)} \lambda^{\alpha-1} \exp (-\beta \lambda)
$$

For $m$ independent counts $n_{\mathrm{i}}$, each Poisson with mean $\lambda t_{\mathrm{i}}$, the posterior density function is

$$
f\left(\lambda \mid n_{1}, \ldots, n_{m}\right)=\frac{\lambda^{\alpha+\sum n_{i}-1} \mathrm{e}^{-\left(\beta+\sum t\right) \lambda}}{\int_{0}^{\infty} \lambda^{\alpha+\sum n_{i}-1} \mathrm{e}^{-\left(\beta+\sum t,\right) \lambda} \mathrm{d} \lambda}
$$

which is gamma( $\alpha+n, \beta+t)$ where $t=\Sigma_{\mathrm{i}} t_{\mathrm{i}}$ and $n=\Sigma_{\mathrm{i}} n_{\mathrm{i}}$.

The noninformative prior, which corresponds to taking $\alpha=1 / 2$ and $\beta=0$, is proportional to $\lambda^{-1 / 2}$, and the posterior distribution for $\lambda$ is gamma $(n+1 / 2, t)$. Another way to express this, which is more convenient for the purpose at hand, is to say that the posterior distribution of $2 t \lambda$ is $\chi^{2}$ with $\nu=2 n+$ 1 degrees of freedom. This uses two well known facts; if $X$ is $\operatorname{gamma}(\alpha, \beta)$, then (1) $X / c$ is $\operatorname{gamma}(\alpha$, $c \beta$ ) for any positive constant $c$, and (2) $2 \beta X$ is $\chi^{2}$ distributed with $\nu=2 \alpha$ degrees of freedom. Consequently, the 0.05 th and 0.95 th quantiles of the posterior distribution of $\lambda$ are $\chi_{0.05}^{2}(2 n+1) /(2 t)$ and $\chi_{0.95}^{2}(2 n+1) /(2 t)$, respectively. These limits clearly differ from the $90 \%$ confidence limits of Equations (B-2) only in the way degrees of freedom are calculated. 


\section{EMPIRICAL BAYES METHOD}

\section{Gamma-Poisson Likelihood}

If the number of observed events $n$ during a period of length $t$, given $\lambda$, has a Poisson $(\lambda t)$ distribution, and if $\lambda$ has a gamma $(\alpha, \beta)$ distribution, then the unconditional distribution of the number of events is called gamma-Poisson $(\alpha, \beta)$. It is a straightforward exercise to show that the probability mass function of the gamma-Poisson distribution is

$$
p(n)=\frac{\Gamma(\alpha+n)}{n ! \Gamma(\alpha)}(t / \beta)^{n}(1+t / \beta)^{-(\alpha+n)} \quad n=0,1,2, \ldots
$$

Now suppose that there are $m$ plants, and that the $i$ th plant has $n_{\mathrm{i}}$ events during a period of length $t_{\mathrm{i}}$. Then the log-likelihood is

$$
\ln L=\sum_{i=1}^{m}\left[\ln \Gamma\left(\alpha+n_{i}\right)-\ln \Gamma(\alpha)-n_{i} \ln (\beta)-\left(\alpha+n_{i}\right) \ln \left(1+t_{i} / \beta\right)\right]
$$

plus an expression that does not depend on $\alpha$ or $\beta$.

\section{Maximum Likelihood Estimation}

The method of maximum likelihood is discussed by Johnson, Kotz, and Kemp (1992) for the case of a random sample. However, in the present application, the observed counts are not identically distributed because different data sources have different exposure times. Thus, it is necessary to derive the MLE's in this more general setting.

To obtain the maximum likelihood estimates $\hat{\alpha}$ and $\hat{\beta}$, the log-likelihood must be maximized numerically. Two ways to do it are mentioned here. A numerical method could be written using the derivatives of $\ln \Gamma$.

$$
\begin{aligned}
& \frac{\partial}{\partial \alpha} \ln L=\sum_{i=1}^{m}\left[\psi\left(\alpha+n_{i}\right)-\psi(\alpha)-\ln \left(1+t_{i} / \beta\right)\right] \\
& \frac{\partial}{\partial \beta} \ln L=-\frac{1}{\beta} \sum_{i=1}^{m}\left[n_{i}-\frac{\alpha+n_{i}}{\beta+t_{i}} t_{i}\right]
\end{aligned}
$$


The first derivative of $\ln \Gamma$, known as the digamma function and denoted by $\psi$, is built into many computer packages, including SAS. Setting these partials to zero and simplifying, we obtain the following maximum likelihood equations

$$
\sum_{i=1}^{m}\left[\psi\left(\hat{\alpha}+n_{j}\right)-\psi(\hat{\alpha})-\ln \left(1+t_{i} / \hat{\beta}\right)\right]=0
$$

$$
\hat{\alpha}=\frac{m \bar{n}-\sum_{i=1}^{m} n_{i}\left(\frac{t_{i}}{\hat{\beta}+t_{i}}\right)}{\sum_{i=1}^{m}\left[\frac{t_{i}}{\hat{\beta}+t_{i}}\right)}
$$

In Equation (B-5), $\hat{\alpha}$ is expressed as a function of $\hat{\beta}$. Substitution of this expression for $\hat{\alpha}$ into Equation (B-4), yields an equation in the variable $\hat{\beta}$. Although this equation cannot be solved explicitly for $\hat{\beta}$, it is an increasing function of $\hat{\beta}$, which can be solved by numerical methods when a positive finite solution exists. Leven and Reeds (1977) proved that when the $t_{\mathrm{i}}$ 's are equal, if at least one $n_{\mathrm{i}}$ is positive, then the likelihood function has at most one local maximum, and the MLE $\hat{\alpha}$ is finite if and only if $s_{\mathrm{N}}{ }^{2}>\bar{n}$. Conditions for $\hat{\alpha}$ to be finite when the $t_{\mathrm{i}}$ 's are not all equal have not been established.

It should be mentioned that it is not necessary to use a programming language with the digamma function built in. An alternative approach is to use the recursive property of the digamma function $\psi(t$ $+1)=\psi(t)+1 / t$. By repeated use of this property, we can obtain that

$$
\psi\left(\hat{\alpha}+n_{i}\right)-\psi(\hat{\alpha})=\sum_{j=1}^{n_{i}} \frac{1}{\hat{\alpha}+j-1}
$$

which can be used to evaluate the left side of equation (B-4).

A program for finding the MLE's called gpmle is given in Appendix A. This program is a macro and it is written in SAS.

\section{Moment Estimation}

A method that is numerically simpler than maximum likelihood is often desirable. Moment estimates are also discussed by Johnson, Kotz and Kemp (1992), but they apply only to the case of a random sample. Thus, a more general method, which applies with unequal exposure times, is required. 
The moment estimators $\alpha^{*}$ and $\beta^{*}$ can be defined, based on the following properties of the mean and variance of gamma-Poisson random variables: If $N_{\mathrm{i}}$ is distributed according to (B-3), then

$$
\begin{aligned}
& \mathrm{E}\left(N_{i}\right)=(\alpha / \beta) t_{i}=\mu t_{i} \\
& \operatorname{var}\left(N_{i}\right)=(\alpha / \beta) t_{i}\left(1+t_{i} / \beta\right)=\mu t_{i}\left(1+t_{i} / \beta\right)
\end{aligned}
$$

The notation $\mu$ is used for $\alpha / \beta$, because $\alpha / \beta$ is the mean of the underlying gamma distribution.

Moment estimators based on the ratio of event counts to exposure times have a very simple form. In particular, if we define $Y_{\mathrm{i}}=N_{\mathrm{i}} / t_{\mathrm{i}}$, for $1 \leq i \leq m$, then $\mathrm{E}\left(Y_{\mathrm{i}}\right)=\mu$ and $\operatorname{var}\left(Y_{\mathrm{i}}\right)=\mu\left(1 / \beta+1 / t_{\mathrm{i}}\right)$. Notice that even though the $Y_{\mathrm{i}}$ 's all have the same mean $\mu$ and they are independent, they do not conform to the usual definition of a random sample when the $t_{\mathrm{i}}$ 's are not all equal, because they have different variances. The standard method of moments cannot be used because it assumes that the data are obtained from a random sample. However, it is possible in this case to use a variation on the standard approach. We proceed by noting that the second moment about zero is $\mathrm{E}\left(Y_{\mathrm{i}}^{2}\right)=\operatorname{var}\left(Y_{\mathrm{i}}\right)+\left[\mathrm{E}\left(Y_{\mathrm{i}}\right)\right]^{2}=\mu^{2}+\mu / \beta+$ $\mu / t_{\mathrm{i}}$. Suppose we now define $\bar{Y}=\Sigma_{\mathrm{i}} Y_{\mathrm{i}} / m$ and $S_{\mathrm{Y}}{ }^{2}=\Sigma_{\mathrm{i}}\left(Y_{\mathrm{i}}-\bar{Y}\right)^{2} /(m-1)$. Then $\mathrm{E}(\bar{Y})=\mu$, and $\operatorname{var}(\bar{Y})=$ $(\mu / m)\left(1 / \beta+\Sigma_{\mathrm{i}} t_{\mathrm{i}}^{-1} / m\right)$. It follows that, $\mathrm{E}\left(\bar{Y}^{2}\right)=\operatorname{var}(\bar{Y})+[\mathrm{E}(\bar{Y})]^{2}=(\mu / m)\left[1 / \beta+\Sigma_{\mathrm{i}} t_{\mathrm{i}}^{-1} / m\right]+\mu^{2}$. Thus, because $(m-1) S_{\mathrm{Y}}{ }^{2}=\Sigma_{\mathrm{i}} Y_{\mathrm{i}}^{2}-, \mathrm{E}\left(S_{\mathrm{Y}}{ }^{2}\right)=\mu\left(1 / \beta+\Sigma_{\mathrm{i}} t_{\mathrm{i}}^{-1} / m\right)$. The moment estimates are obtained by equating the estimates $\bar{y}$ and $s_{Y}{ }^{2}$ to their expected values and solving for $\mu^{*}$ and $\beta^{*}$. In particular, we get

$$
\begin{aligned}
& \mu^{*}=\bar{y} \\
& \beta^{*}=\left[\frac{s_{Y}^{2}}{\bar{y}}-\frac{1}{m} \sum_{i=1}^{m} t_{i}^{-1}\right]^{-1}
\end{aligned}
$$

Note that $\beta^{*}>0$ if and only if $s_{\mathrm{Y}}{ }^{2} / \bar{y}>\Sigma_{\mathrm{i}} t_{\mathrm{i}}{ }^{-1} / m$. The moment estimator of $\alpha$ is $\alpha^{*}=\mu^{*} \beta^{*}$. As noted above, the values obtained can be negative for some sets of data, but they do not require an iterative numerical method. In a limited number of comparisons, the moment estimates were found to be numerically close to the MLE's. The moment estimates provide starting values for the iterative solution of the maximum likelihood equations. 


\section{Adjustment for Uncertainty in Maximum Likelihood Estimates}

Kass and Steffey (1989) present an adjustment to account for the fact that the parameters $\alpha$ and $\beta$ are only estimated from data, and not known exactly. The method makes use of the variance and covariances of the estimators. Kass and Steffey state that the adjustment is very important if there are few data sources (such as plants) and many observations, and conversely that the adjustment is unimportant when there are many data sources and few observations. The SAS macro gpempb makes the adjustment automatically, because it is computationally quick.

Atwood (1984) points out both theoretical and computational advantages in parameterizing in terms of $\alpha$ and $\mu=\alpha / \beta$. Specifically, for maximum likelihood estimators, the variance-covariance matrix is estimated by inverting the sample information matrix, that is, the negative of the matrix of second derivatives of the log-likelihood. When parameterized by $\alpha$ and $\mu$, the sample information matrix and consequently, the variance-covariance matrix are diagonal matrices. In other words, the MLEs $\hat{\alpha}$ and $\hat{\mu}$ are asymptotically uncorrelated. This not only simplifies the mathematics involved in expressing the asymptotic variances, but it also has computational benefits.

Denote by $\lambda_{\mathrm{i}}$ the rate of occurrence at the $i$ th plant. We wish to find the posterior mean and variance of $\lambda_{\mathrm{i}}$. We denote the vectors of counts and exposure times as $\mathbf{n}=\left(n_{1}, n_{2}, \ldots, n_{\mathrm{m}}\right)$ and $\mathbf{t}=\left(t_{1}\right.$, $\left.t_{2}, \ldots, t_{\mathrm{m}}\right)$. The posterior mean and variance denoted by $E\left(\lambda_{i} \mid \mathbf{n} ; \mathbf{t}\right)$ and $\operatorname{var}\left(\lambda_{\mathrm{i}} \mid \mathbf{n} ; \mathbf{t}\right)$, are approximated by

$$
\begin{aligned}
& E\left(\lambda_{i} \mid n_{i} ; \hat{\alpha}, \hat{\mu}, t_{i}\right)=\frac{\hat{\alpha}+n_{i}}{\hat{\alpha} / \hat{\mu}+t_{i}}, \\
& \operatorname{var}\left(\lambda_{i} \mid n_{i} ; \hat{\alpha}, \hat{\mu}, t_{i}\right)=\frac{\hat{\alpha}+n_{i}}{\left(\hat{\alpha} / \hat{\mu}+t_{i}\right)^{2}} .
\end{aligned}
$$

where $\hat{\mu}=\hat{\alpha} / \hat{\beta}$.

The Kass-Steffey adjustment estimates the posterior mean for the $i$ th data source by $E_{\mathrm{i}}=E\left(\lambda_{\mathrm{i}} \mid n_{\mathrm{i}}\right.$; $\hat{\alpha}, \hat{\mu}, t_{\mathrm{i}}$ ), but it increases the variance estimate to

$$
\operatorname{var}\left(\lambda_{i} \mid n_{i}, \hat{\alpha}, \hat{\mu}\right)+\left[\frac{\partial E_{i}}{\partial \alpha}\right]^{2} \operatorname{var}(\hat{\alpha})+\left[\frac{\partial E_{i}}{\partial \mu}\right]^{2} \operatorname{var}(\hat{\mu})
$$


where the partials are evaluated at $\alpha=\hat{\alpha}$ and $\mu=\hat{\mu}$. In general, a covariance term is also present, but in this application the MLEs $\hat{\alpha}$ and $\hat{\mu}$ are asymptotically uncorrelated, so the covariance term is zero. The variance terms are estimated by evaluating the information matrix at $\hat{\alpha}$ and $\hat{\mu}$. The inverse of this matrix is the estimated variance-covariance matrix of $(\hat{\alpha}, \hat{\mu})$. The estimated variances $\operatorname{var}(\hat{\alpha}) \operatorname{and} \operatorname{var}(\hat{\mu})$ are found by the SAS macro gpmle.

The Kass-Steffey adjustment concludes by finding the values of $\alpha$ and $\beta$ that correspond to the estimated posterior mean and variance. These values are used to define the posterior distribution of $\lambda_{i}$, modeled as a gamma distribution. The SAS macro gpempb performs these calculations, and finds the empirical Bayes $90 \%$ interval for each $\lambda_{\mathrm{i}}$. 


\section{REFERENCES}

Atwood, Corwin L., 1984, “Approximate Tolerance Intervals, Based on Maximum Likelihood Estimates," Technometrics, 79, pp. 459-465.

Atwood, Corwin L., 1994, Hits per Trial: Basic Analysis of Binomial Data, EGG-RAAM-11041.

Bain, Lee J., and Max Engelhardt, 1992, Introduction to Probability and Mathematical Statistics, Boston: PWS-Kent.

Kass, Robert E. and Duane Steffey, 1989, “Approximate Bayesian Inference in Conditionally Independent Hierarchical Models (Parametric Empirical Bayes Models)," Journal of the American Statistical Association, 84, pp. 717-726.

Leven, B., and Reeds, J., 1977, "Compound Multinomial Likelihood Functions are Unimodal," Annals of Statistics, 5, 79-87. 